\title{
Photometry and Spectroscopy of Faint Candidate Spectrophotometric Standard DA White Dwarfs
}

\author{
Annalisa Calamida $^{1,2}$ (D) Thomas Matheson $^{1}$ (D), Abhijit Saha ${ }^{1}$ (D), Edward Olszewski ${ }^{3}$, Gautham Narayan ${ }^{1,2}$ (D), Jenna Claver ${ }^{1}$, \\ Clare Shanahan $^{2}$, Jay Holberg ${ }^{4}$, Tim Axelrod $^{3}$ (D), Ralph Bohlin ${ }^{2}$ (D), Christopher W. Stubbs ${ }^{5}$ (D), Susana Deustua ${ }^{2}$ (iD, Ivan Hubeny ${ }^{3}$, \\ John Mackenty ${ }^{2}$, Sean Points ${ }^{6}$, Armin Rest ${ }^{2,7}$, and Elena Sabbi ${ }^{2}$ (i) \\ ${ }^{1}$ National Optical Astronomy Observatory-AURA, 950 N Cherry Avenue, Tucson, AZ 85719, USA; calamida@stsci.edu \\ ${ }^{2}$ Space Telescope Science Institute-AURA, 3700 San Martin Drive, Baltimore, MD 21218, USA \\ ${ }^{3}$ Steward Observatory, University of Arizona, 933 N Cherry Avenue, Tucson, AZ 85719, USA \\ ${ }^{4}$ Lunar and Planetary Laboratory, University of Arizona, 1629 E University Boulevard, Tucson, AZ 85721, USA \\ ${ }_{5}^{5}$ Harvard University, 17 Oxford Street, Cambridge, MA 02138, USA \\ ${ }^{6}$ Cerro Tololo Inter-American Observatory, Casilla 603, La Serena, Chile \\ ${ }^{7}$ Department of Physics and Astronomy, Johns Hopkins University, Baltimore, MD 21218, USA \\ Received 2018 November 13; revised 2018 December 22; accepted 2018 December 23; published 2019 February 25
}

\begin{abstract}
We present precise photometry and spectroscopy for 23 candidate spectrophotometric standard white dwarfs. The selected stars are distributed in the Northern hemisphere and around the celestial equator, and are all fainter than $r \sim 16.5 \mathrm{mag}$. This network of stars, when established as standards and together with the three Hubble Space Telescope primary CALSPEC white dwarfs, will provide a set of spectrophotometric standards to directly calibrate data products to better than $1 \%$. In future deep photometric surveys and facilities, these new faint standard white dwarfs will have enough signal-to-noise ratio to be measured accurately while still avoiding saturation. They will also fall within the dynamic range of large telescopes and their instruments for the foreseeable future. This paper discusses the provenance of the observational data for our candidate standard stars. A comparison with models, reconciliation with reddening, and the consequent derivation of the full spectral energy density distributions for each of them is reserved for a subsequent paper.
\end{abstract}

Key words: methods: observational - standards - stars: fundamental parameters - techniques: photometric techniques: spectroscopic - white dwarfs

Supporting material: machine-readable tables

\section{Introduction}

Astrophysics is at the threshold of an era of deep imaging surveys of large portions of the sky, both from the ground and from telescopes in space. Projects such as the Sloan Digital Sky Survey (SDSS), Pan-STARRS (PS), the Dark Energy Survey (DES), Skymapper, the Asteroid Terrestrial-impact Last Alert System (ATLAS), the All Sky Automated Survey for SuperNovae (ASAS-SN), the Galaxy Evolution Explorer (GALEX), and the Wide-field Infrared Survey Explorer (WISE) are either complete or in their advanced stages, while the Zwicky Transient Facility (ZTF) has just begun and the Large Synoptic Survey Telescope (LSST) is only a few years away. ${ }^{8}$ GAIA and Kepler continue to report photometry of millions of stars to very high internal accuracy. These projects have their own native pass-bands and photometric systems, some of which are similar while others differ significantly. To make the information across these projects commensurate with each other, we must put them on a common photometric system. Therefore, we need a set of calibration references, with which both existing and future surveys can be cross-calibrated. Specifically, we seek to establish a set of standard stars that satisfy the following criteria:

1. They must have relative spectral energy distributions (SEDs) that are established to sub-percent accuracy, and preferably to better than half-percent.

\footnotetext{
8 Table 10 in the Appendix lists and describes all the acronyms used in the manuscript.
}

2. They must fall within the dynamic range of most, if not all, extant and future deep surveys. We surmise that these stars should be fainter than $r \sim 16.5 \mathrm{mag}$, which also puts them within the dynamic range of large largeaperture telescopes.

3. They must be distributed across the sky so that they are naturally observed in past, present and future surveys, making it possible to retroactively re-calibrate photometry onto a common (spectro)-photometric scale. This will allow the direct collation of photometry from different surveys with their own respective native passbands onto a commensurate platform. For other investigations, a few of the standards will always be available from any observatory at any point in time.

Sub-percent global photometric standardization has proven challenging in the past but is in high demand for several ongoing scientific endeavors. It is also the major source of uncertainty in the use of Type Ia supernovae as probes of the history of cosmic expansion to infer the properties of dark energy (Betoule et al. 2014; Scolnic et al. 2015; Stubbs \& Brown 2015). Experiments that require accurate and reliable photoredshift determination, such as weak lensing tomography and baryonic acoustic oscillation analysis with LSST (Gorecki et al. 2014), are also limited by systematic uncertainties arising from their relative photometric calibration.

The chief obstacle for calibrating standard stars with high accuracy from the ground by comparing them to laboratory sources is the uncertainty in atmospheric extinction. Ground-based 
survey accuracy is limited by the transmissivity of the atmosphere, with both chromatic (Rayleigh scattering, ozone, Mie scattering, molecular absorption, aerosol) and gray (clouds) terms varying on small angular and temporal scales. A variety of methods are used to track and account for these effects, including monitoring (e.g., LIDAR, GPS). Many efforts are also made to model the atmosphere (e.g., with MODTRAN, Burke et al. 2014). Although it would be ideal to place laboratory sources above the terrestrial atmosphere, this is unlikely to happen in the near future. For a more detailed discussion of the problems related to obtaining a sub-percent accurate calibration, please see Narayan et al. (2016, hereafter NA16).

Therefore, we seek extra-terrestrial sources for which we can predict the SED to higher accuracy than the uncertainty in predicting the transmissivity of the terrestrial atmosphere. The best class of celestial objects that we can hope to characterize and model are hot DA white dwarfs (DAWDs). These stars have almost pure-hydrogen atmospheres, which makes them the simplest stellar atmospheres to model. In addition, their opacities are known from first principles, at temperatures greater than $\sim 20,000 \mathrm{~K}$ the photospheres are purely radiative, and they are photometrically stable.

The intrinsic DAWD SED can be described by two parameters: effective temperature, $T_{\text {eff }}$, and surface gravity, $\log (g)$. Both of these parameters can be measured spectroscopically from a detailed analysis of the Balmer line profiles, without using photometry. The SED can then be modeled from the ultraviolet (UV) to the near-infrared and projected through the transmission of any imager or spectrometer at arbitrary resolution. Only the extinction toward the observed DAWDs and the overall flux normalization need to be established.

Bohlin (2000) and Bohlin et al. (2014, hereafter B14) implemented the pure-hydrogen-WD method of flux calibration using three DAWDs-G191B2B, GD153 and GD71 (Hubble Space Telescope (HST) primary WDs). These stars are brighter than $V \sim 13.5 \mathrm{mag}$, they span a range of temperatures $30,000 \lesssim T_{\text {eff }} \lesssim 60,000$, and they are un-reddened as a result of their proximity to us. B14 found their relative flux distributions to be internally consistent with the model predictions (Gianninas et al. 2011; Rauch et al. 2013) from spectroscopic $T_{\text {eff }}$ and $\log g$ to better than $1 \%$ in the wavelength range $0.2-0.9 \mu \mathrm{m}$. Spectrophotometry of Vega with STIS (Bohlin \& Gilliland 2004; Bohlin 2007) referred to the DAWD flux scale shows agreement with Hayes et al.'s (1985) calibration at the $1 \%-2 \%$ level, and with the Kurucz ${ }^{9}$ Vega atmosphere model to better than $1 \%$ in the wavelength range $0.5-0.8 \mu \mathrm{m}$, but disagrees by $5 \%$ at $0.4 \mu \mathrm{m}$, and by $10 \%$ between 0.9 and $1.0 \mu \mathrm{m}$. This illustrates the limitation of empirical ground-based methods.

The internal consistency of the DAWD observations and models ( $\leqslant 5 \mathrm{mmag}$ ) in the wavelength range $0.2-0.9 \mu \mathrm{m}$ is superior to the $\sim 2 \%$ comparison with the best model for Vega (Kurucz at $T_{\text {eff }}=9400 \mathrm{~K}$ ), which is a pole-on rapid rotator with an equatorial dust disk. The zero-point (ZP) for the HST photometric system is defined by the flux of $3.44 \times$ $10^{-9} \mathrm{erg} \mathrm{cm}^{-2} \mathrm{~s}^{-1} \AA^{-1}$ for Vega at $0.5556 \mu \mathrm{m}$, as reconciled with the Midcourse Space Experiment (MSX) mid-IR absolute flux measures (B14, and Bohlin 2014). Absolute fluxes for the three HST primary WDs are determined by the normalization of their modeled SEDs by their respective relative responses to

\footnotetext{
9 http://kurucz.harvard.edu/stars/vega/
}

Vega, using STIS precision spectrophotometry of all four stars -Vega, G191B2B, GD153 and GD71-and the $3.44 \times$ $10^{-9} \mathrm{erg} \mathrm{cm}^{-2} \mathrm{~s}^{-1} \AA^{-1}$ flux of Vega at $0.5556 \mu \mathrm{m}$. This method provides the basis for HST's entire calibration system (CALSPEC ${ }^{10}$ ).

Holberg \& Bergeron (2006) used synthetic photometry of DAWDs in the magnitude range $10 \lesssim V \lesssim 16.5$ to place UBVRI, 2MASS JHK, SDSS ugriz and Strömgren ubvy magnitudes on the HST photometric scale to $1 \%$. Later, Holberg et al. (2008) confirmed this calibration by using a set of DAWDs in the same magnitude range with well-measured trigonometric parallaxes that agreed at the $1 \%$ level with their photometric parallaxes from the Bergeron photometric grid. However, the DAWDs in use to date are still too bright for modern deep surveys and large telescopes.

To provide flux standards in the dynamic range of largeaperture $(d>4 \mathrm{~m})$ telescopes, we obtained Wide-Field Camera 3 (WFC3) HST imaging and ground-based spectroscopy for the three HST primary (CALSPEC) standards-G191B2B, GD153, and GD71-along with 23 DAWDs fainter than $r \sim 16.5 \mathrm{mag}$, at equatorial and northern latitudes. The need for practical faint standards, which are useful over the optical and near-UV, makes consideration of the effects of interstellar extinction unavoidable. Indeed, interstellar medium extinction must be incorporated into the definition of the SEDs of all faint flux standards. Fortunately, as sub-luminous stars, DAWDs are the optimal choice because they simultaneously offer minimal extinction columns and wide wavelength coverage, from the far-UV to the IR.

The current paper presents our analysis of photometric and spectroscopic data collected for the candidate spectrophotometric DAWDs. The preliminary results of the temporal photometric monitoring campaign of the DAWDs are also presented. Photometric and spectroscopic data are examined to determine the suitability of each of the 23 candidates as SED standards. The joint analysis of photometry and spectroscopy and the derivation of SEDs and reddening to each of these objects are reserved for a subsequent paper (Narayan et al. 2019, hereafter NA19).

The structure of the current paper is as follows. In Section 2, we discuss the criteria used to select candidate spectrophotometric standard DAWDs and in Section 3 we illustrate the photometric observations and the image processing strategy. In Section 4, we describe the photometric reduction procedures and in Section 5 the stability monitoring observations for the candidate standards. In Section 6, the spectroscopic data reduction strategy is described and in Section 7 we discuss how our photometry is calibrated and normalized. We summarize our results in Section 8.

\section{Candidate Spectrophotometric Standard Star Selection}

Candidate spectrophotometric standard DAWDs were selected from the SDSS (Adelman-McCarthy et al. 2008; Girven et al. 2012; Kleinman et al. 2013) and the Villanova catalog (McCook \& Sion 1999), with the requirement of being spectral type DA, hotter than $\approx 20,000 \mathrm{~K}$, and fainter than $\mathrm{r} \sim 16.5 \mathrm{mag}$. We selected an adequate number of stars to uniformly cover the sky around the celestial equator and in the Northern hemisphere. The final sample consists of 23 candidate standard DAWDs. Table 1 lists the properties of the selected

\footnotetext{
${ }^{10}$ http://www.stsci.edu/hst/observatory/crds/calspec.html
} 


\begin{tabular}{|c|c|c|c|c|c|c|c|c|c|}
\hline Star & Alt Name & $\begin{array}{c}\text { R.A. }^{\mathrm{a}} \\
\text { (hh:mm:ss.s) }\end{array}$ & $\begin{array}{c}\text { Decl. }^{\mathrm{a}} \\
\text { (dd:mm:ss.s) }\end{array}$ & 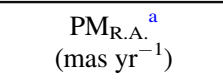 & $\begin{array}{l}\mathrm{PM}_{\text {Decl.1. }}{ }^{\mathrm{a}} \\
\left(\mathrm{mas} \mathrm{yr}^{-1}\right)\end{array}$ & SType $^{\mathrm{a}}$ & $\begin{array}{l}\text { Distance }^{\mathrm{a}} \\
(\mathrm{pc})\end{array}$ & $\begin{array}{l}T_{\text {efff }}^{\mathrm{b}} \\
(\mathrm{K})\end{array}$ & $\log (g)^{\mathrm{b}}$ \\
\hline G191B2B & $\mathrm{BD}+52913$ & $5: 05: 30.613$ & $52: 49: 51.956$ & $12.592 \pm 0.122$ & $-93.525 \pm 0.106$ & DA. 89 & $52.9 \pm 0.2$ & 57340 & 7.48 \\
\hline GD71 & GD71 & $5: 52: 27.614$ & $15: 53: 13.751$ & $76.841 \pm 0.131$ & $-172.944 \pm 0.104$ & DA1.5 & $51.9 \pm 0.2$ & 32780 & 7.83 \\
\hline GD153 & GD153 & 12:57:02.337 & $22: 01: 52.68$ & $-38.410 \pm 0.119$ & $-202.953 \pm 0.116$ & DA1.3 & $68.6 \pm 0.3$ & 39390 & 7.77 \\
\hline SDSSJ010322.19-002047.7 & SDSSJ010322.19-002047.7 & 1:03:22.191 & $-0: 20: 47.731$ & $6.216 \pm 0.957$ & $-6.313 \pm 0.549$ & DA.67 & $1097 \pm 611$ & 75000 & 7.81 \\
\hline SDSSJ022817.16-082716.4 & WD0225-086 & 2:28:17.169 & $-8: 27: 16.409$ & $12.241 \pm 1.461$ & $3.827 \pm 1.000$ & DA2.45 & $525 \pm 181$ & 20555 & 7.87 \\
\hline SDSSJ024854.96+334548.3 & SDSSJ024854.96+334548.3 & $2: 48: 54.967$ & $33: 45: 48.33$ & $3.635 \pm 0.700$ & $-4.718 \pm 0.442$ & DA1.46 & $630 \pm 128$ & 34497 & 7.30 \\
\hline SDSSJ041053.632-063027.580 & WD0408-066 & $4: 10: 53.634$ & $-6: 30: 27.749$ & $8.620 \pm 0.411$ & $9.700 \pm 0.237$ & DA.77 & $1833 \pm 1248$ & 65796 & 7.52 \\
\hline WD0554-165 & WD0554-165 & 5:57:01.296 & $-16: 35: 12.12$ & $-7.188 \pm 0.399$ & $4.781 \pm 0.623$ & . & $239 \pm 13$ & & \\
\hline SDSSJ072752.76+321416.1 & SDSSJ072752.76+321416.1 & 7:27:52.76 & 32:14:16.141 & $-13.095 \pm 0.366$ & $-7.094 \pm 0.373$ & DA. 88 & $990 \pm 198$ & 57865 & 7.61 \\
\hline SDSSJ081508.78+073145.7 & SDSSJ081508.78+073145.7 & $8: 15: 08.779$ & 7:31:45.804 & $3.135 \pm 1.384$ & $0.313 \pm 0.794$ & DA1.55 & $\ldots$ & 32387 & 6.81 \\
\hline SDSSJ102430.93-003207.0 & SDSSJ102430.93-003207.0 & 10:24:30.932 & $-0: 32: 07.03$ & -24.0 & -5.0 & DA1.21 & . & 41584 & 7.77 \\
\hline SDSSJ111059.42-170954.2 & SDSSJ111059.42-170954.2 & $11: 10: 59.428$ & $-17: 09: 54.27$ & $5.045 \pm 0.418$ & $-7.763 \pm 0.293$ & DA.96 & $1333 \pm 359$ & 52555 & 7.73 \\
\hline SDSSJ111127.30+395628.0 & SDSSJ111127.30+395628.0 & 11:11:27.309 & 39:56:28.079 & $3.277 \pm 0.445$ & $3.095 \pm 0.598$ & DA.75 & $648 \pm 166$ & 67380 & 7.80 \\
\hline SDSSJ120650.504+020143.810 & WD1204+023 & 12:06:50.408 & $2: 01: 42.46$ & $-4.594 \pm 0.663$ & $-23.143 \pm 0.319$ & DA2.02 & $590 \pm 130$ & 24926 & 7.98 \\
\hline SDSSJ121405.11+453818.5 & CSO1291 & 12:14:05.112 & $45: 38: 18.56$ & $0.291 \pm 0.140$ & $13.803 \pm 0.170$ & DA1.43 & $495 \pm 31$ & 35245 & 7.91 \\
\hline SDSSJ130234.43+101238.9 & SDSSJ130234.43+101238.9 & 13:02:34.441 & $10: 12: 39.01$ & $-12.523 \pm 0.252$ & $-17.372 \pm 0.191$ & DA1.20 & $389 \pm 17$ & 42070 & 7.91 \\
\hline SDSSJ131445.050-031415.588 & WD1312-029 & $13: 14: 45.05$ & $-3: 14: 15.641$ & $-4.102 \pm 1.190$ & $-6.354 \pm 0.606$ & DA1.05 & $1154 \pm 834$ & 47818 & 7.76 \\
\hline SDSSJ151421.27+004752.8 & LB 769 & $15: 14: 21.28$ & $0: 47: 52.883$ & $4.400 \pm 0.175$ & $-27.041 \pm 0.222$ & DA1.74 & $157 \pm 3$ & 28999 & 7.81 \\
\hline SDSSJ155745.40+554609.7 & WD1556+559 & $15: 57: 45.404$ & $55: 46: 09.75$ & $-11.545 \pm 0.260$ & $-21.340 \pm 0.204$ & DA.79 & $688 \pm 52$ & 64122 & 7.65 \\
\hline SDSSJ163800.360+004717.822 & WD1635+008 & 16:38:00.366 & $0: 47: 17.801$ & $-9.582 \pm 0.782$ & $-2.797 \pm 0.467$ & DA.77 & $876 \pm 283$ & 65116 & 7.37 \\
\hline SDSSJ172135.97+294016.0 & SDSSJ172135.97+294016.0 & $17: 21: 35.981$ & 29:40:15.996 & $-21.454 \pm 0.564$ & $10.452 \pm 0.638$ & DA5.44 & $271 \pm 26$ & 9261 & 8.33 \\
\hline SDSSJ181424.075+785403.048 & WD1817+788 & 18:14:24.122 & 78:54:02.909 & $-11.041 \pm 0.103$ & $11.292 \pm 0.132$ & DA1.6 & $257 \pm 3$ & 31500 & 7.81 \\
\hline SDSSJ20372.169-051302.964 & WD2034-053 & 20:37:22.167 & $-5: 13: 03.029$ & $3.106 \pm 0.647$ & $-2.723 \pm 0.389$ & DA1.33 & $912 \pm 324$ & 37923 & 7.92 \\
\hline SDSSJ210150.65-054550.9 & WD2059-059 & 21:01:50.657 & $-5: 45: 50.969$ & $10.828 \pm 0.456$ & $-11.727 \pm 0.372$ & DA1.75 & $662 \pm 107$ & 28816 & 7.78 \\
\hline SDSSJ232941.330+001107.755 & WD2327-000 & 23:29:41.325 & 0:11:07.8 & $-8.299 \pm 0.384$ & $-14.421 \pm 0.277$ & DA2.37 & $318 \pm 25$ & 21266 & 7.88 \\
\hline SDSSJ235144.29+375542.6 & SDSSJ235144.29+375542.6 & $23: 51: 44.293$ & $37: 55: 42.661$ & $-16.575 \pm 0.294$ & $-10.048 \pm 0.185$ & DA.95 & $765 \pm 134$ & 53333 & 7.72 \\
\hline
\end{tabular}

Notes.

Coordinates, proper motions, spectral type, and distance measurements are from GAIA DR2.

${ }^{\mathrm{b}}$ Effective temperature and surface gravity measurements are from the SDSS or the Villanova catalogs. 


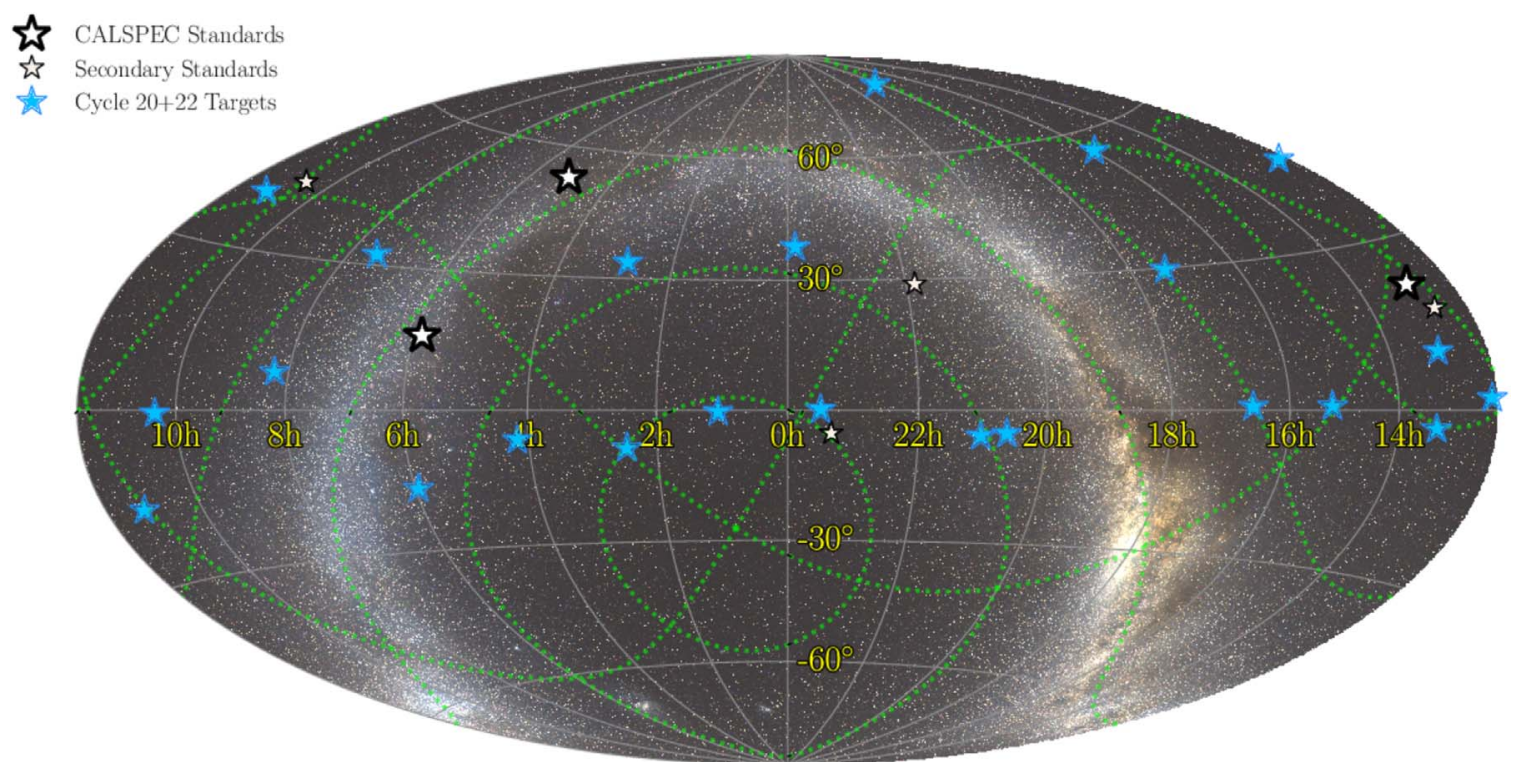

Figure 1. A Hammer-Aitoff projection of the 23 candidate spectrophotometric standard DAWDs observed with $H S T$ in cycles 20 and 22 in equatorial coordinates (blue stars). The three HST primary CALSPEC standards are marked with a large white star and four spectroscopic flux standards (secondary standards) are marked with a small white star.

stars and the three $H S T$ primary CALSPEC standards (GD71, GD153, G191B2B), including spectral type, proper motions and distances from the GAIA data release 2 (DR2, Gaia Collaboration et al. 2018).

Figure 1 shows a Hammer-Aitoff projection of the sky with the distribution of the HST primary (CALSPEC) standard WDs (large white stars) and the 23 selected candidate standard DAWDs (blue). The four secondary flux standard stars that are used to calibrate spectra analyzed in the current paper are also shown in the figure as small white stars. The figure shows that candidate standards have an homogeneous coverage over the Northern hemisphere and the celestial equator, with approximately 1 star every $2 \mathrm{hr}$.

We have a sample of candidate DAWDs for the Southern hemisphere, for which spectra collected with the Goodman spectrograph on the SOAR telescope (CTIO) are available and HST photometry was collected during Cycle 25 (GO-15113, PI: Saha). A subsequent paper will present photometry and spectroscopy for these new candidates. The final goal is to provide an all-sky set of sub-percent precision spectrophotometric standards so that at least three of these stars are visible at any time from any observatory at an airmass less than 2 .

Preliminary effective temperatures and gravities to select candidate spectrophotometric standards were retrieved from the SDSS and the Villanova catalogs. The ground-based spectra that we collected using different facilities will be used to derive more accurate temperatures and gravities for all the DAWDs, as described in Section 6. The HST primary WDs and the candidate standards are plotted in the $T_{\text {eff }}$ versus $\log (g)$ plane in Figure 2. For star WD0554-165, $T_{\text {eff }}$ and $\log (g)$ measurements are unavailable in the literature. The figure shows that star SDSSJ172135.97+294016.0 is much cooler compared to the other DAWDs, with an effective temperature of $T_{\text {eff }}=9261 \mathrm{~K}$ (see Table 1). This star was included in the sample because of an early decision that was made before we restricted ourselves to purely radiative atmospheres with temperatures $T_{\text {eff }}>20,000 \mathrm{~K}$. Although the observations and data reduction for this star were carried through, this object will no longer be included in the network of standard DAWDs.

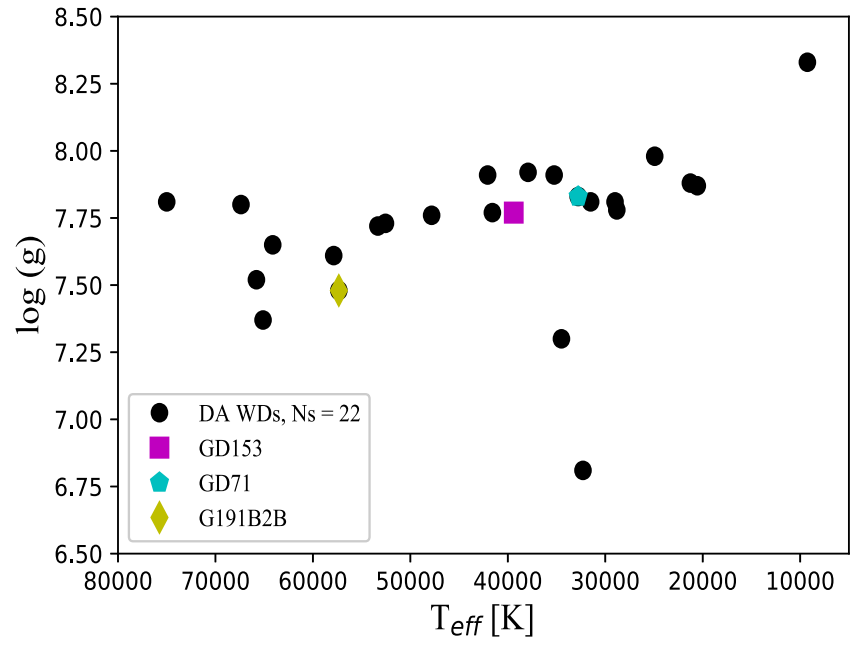

Figure 2. $T_{\text {eff }}$ vs. $\log (g)$ plane for 22 selected DA white dwarfs (black dots) and the three HST primary CALSPEC standards: G191B2B (yellow diamond), GD153 (magenta square), and GD71 (cyan pentagon).

We matched the list of candidates with PS Data Release 1 catalog (Chambers et al. 2016; Flewelling et al. 2016) and obtained $g, r, i, z$ aperture photometry magnitudes for all of these stars. These data will be later used to check the the stellar density around the candidate standards. We also retrieved GAIA DR2 $G, B p, R p$ magnitudes for the DAWDs. PS and GAIA sets of magnitudes are listed in Tables 2 and 3, respectively.

Figure 3 shows the $B p-R p, \mathrm{~F} 475 \mathrm{~W}-\mathrm{F} 775 \mathrm{~W}$ color-color diagram for the 23 candidate standard DAWDs (black dots) and the three primary standards: G191B2B (yellow diamond), GD153 (magenta square) and GD71 (cyan pentagon). The photometry in the HST filters is from this work (see Section 7 and Table 9). The selected candidate standards and the primary HST WDs cluster along a well-defined sequence and have colors in the range $-0.6 \lesssim B p-R p \lesssim-0.1$ and $-0.4 \lesssim$ F475W - F775W $\lesssim 0$ mag, excluding star SDSSJ172135.97 $+294016.0, \sim 0.3-0.4 \mathrm{mag}$ redder than the rest of the WDs, 
Table 2

Pan-STARRS1 Photometry for the Candidate Spectrophotometric Standard DA White Dwarfs

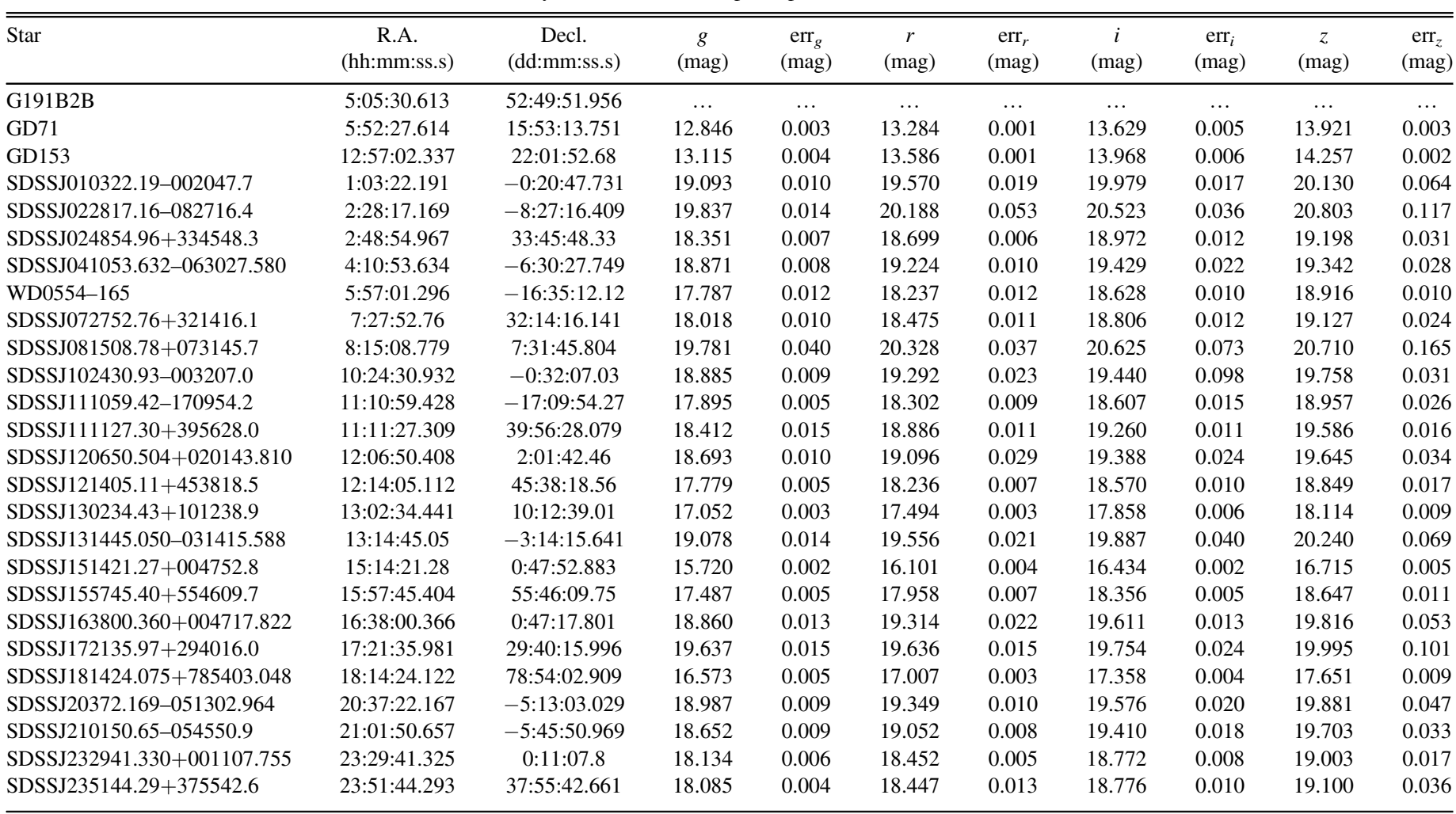

Table 3

GAIA DR2 Photometry for the Candidate Spectrophotometric Standard DA White Dwarfs

\begin{tabular}{|c|c|c|c|c|c|c|c|c|}
\hline Star & $\begin{array}{c}\text { R.A. } \\
\text { (hh:mm:ss.s) }\end{array}$ & $\begin{array}{c}\text { Decl. } \\
\text { (dd:mm:ss.s) }\end{array}$ & $\begin{array}{c}G \\
(\mathrm{mag})\end{array}$ & $\begin{array}{c}\operatorname{err}_{G} \\
(\mathrm{mag})\end{array}$ & $\begin{array}{c}R p \\
(\mathrm{mag})\end{array}$ & $\begin{array}{l}\operatorname{err}_{R p} \\
(\mathrm{mag})\end{array}$ & $\begin{array}{c}B p \\
(\mathrm{mag})\end{array}$ & $\begin{array}{l}\operatorname{err}_{B p} \\
(\mathrm{mag})\end{array}$ \\
\hline G191B2B & $5: 05: 30.613$ & $52: 49: 51.956$ & 11.738 & 0.001 & 12.067 & 0.002 & 11.487 & 0.015 \\
\hline GD71 & $5: 52: 27.614$ & $15: 53: 13.751$ & 13.026 & 0.002 & 13.299 & 0.002 & 12.77 & 0.012 \\
\hline GD153 & $12: 57: 02.337$ & 22:01:52.68 & 13.322 & 0.0 & 13.629 & 0.001 & 13.081 & 0.005 \\
\hline SDSSJ010322.19-002047.7 & 1:03:22.191 & $-0: 20: 47.731$ & 19.356 & 0.004 & 19.577 & 0.072 & 19.154 & 0.03 \\
\hline SDSSJ022817.16-082716.4 & 2:28:17.169 & $-8: 27: 16.409$ & 20.046 & 0.01 & 20.192 & 0.141 & 19.869 & 0.139 \\
\hline SDSSJ024854.96+334548.3 & $2: 48: 54.967$ & $33: 45: 48.33$ & 18.561 & 0.003 & 18.704 & 0.031 & 18.333 & 0.047 \\
\hline SDSSJ041053.632-063027.580 & $4: 10: 53.634$ & $-6: 30: 27.749$ & 19.024 & 0.002 & 19.013 & 0.023 & 18.861 & 0.025 \\
\hline WD0554-165 & $5: 57: 01.296$ & $-16: 35: 12.12$ & 17.98 & 0.003 & 18.306 & 0.03 & 17.726 & 0.022 \\
\hline SDSSJ072752.76+321416.1 & $7: 27: 52.76$ & $32: 14: 16.141$ & 18.232 & 0.003 & 18.458 & 0.036 & 17.944 & 0.007 \\
\hline SDSSJ081508.78+073145.7 & 8:15:08.779 & $7: 31: 45.804$ & 19.996 & 0.005 & 20.278 & 0.166 & 19.695 & 0.044 \\
\hline SDSSJ102430.93-003207.0 & 10:24:30.932 & $-0: 32: 07.03$ & 19.12 & 0.005 & 19.297 & 0.105 & 18.94 & 0.059 \\
\hline SDSSJ111059.42-170954.2 & $11: 10: 59.428$ & $-17: 09: 54.27$ & 18.089 & 0.002 & 18.347 & 0.02 & 17.852 & 0.011 \\
\hline SDSSJ111127.30+395628.0 & 11:11:27.309 & $39: 56: 28.079$ & 18.69 & 0.003 & 18.955 & 0.075 & 18.365 & 0.022 \\
\hline SDSSJ120650.504+020143.810 & $12: 06: 50.408$ & $2: 01: 42.46$ & 18.885 & 0.002 & 18.957 & 0.03 & 18.651 & 0.017 \\
\hline SDSSJ121405.11+453818.5 & $12: 14: 05.112$ & $45: 38: 18.56$ & 18.002 & 0.001 & 18.154 & 0.038 & 17.757 & 0.011 \\
\hline SDSSJ130234.43+101238.9 & $13: 02: 34.441$ & 10:12:39.01 & 17.268 & 0.001 & 17.527 & 0.012 & 17.044 & 0.006 \\
\hline SDSSJ131445.050-031415.588 & $13: 14: 45.05$ & $-3: 14: 15.641$ & 19.354 & 0.004 & 19.631 & 0.082 & 19.082 & 0.042 \\
\hline SDSSJ151421.27+004752.8 & $15: 14: 21.28$ & $0: 47: 52.883$ & 15.905 & 0.001 & 16.119 & 0.005 & 15.743 & 0.009 \\
\hline SDSSJ155745.40+554609.7 & $15: 57: 45.404$ & $55: 46: 09.75$ & 17.721 & 0.002 & 18.019 & 0.018 & 17.452 & 0.014 \\
\hline SDSSJ163800.360+004717.822 & $16: 38: 00.366$ & $0: 47: 17.801$ & 19.065 & 0.002 & 19.313 & 0.04 & 18.853 & 0.019 \\
\hline SDSSJ172135.97+294016.0 & $17: 21: 35.981$ & $29: 40: 15.996$ & 19.648 & 0.005 & 19.528 & 0.037 & 19.733 & 0.042 \\
\hline SDSSJ181424.075+785403.048 & $18: 14: 24.122$ & 78:54:02.909 & 16.773 & 0.002 & 17.031 & 0.007 & 16.57 & 0.009 \\
\hline SDSSJ20372.169-051302.964 & 20:37:22.167 & $-5: 13: 03.029$ & 19.148 & 0.003 & 19.375 & 0.055 & 18.982 & 0.018 \\
\hline SDSSJ210150.65-054550.9 & $21: 01: 50.657$ & $-5: 45: 50.969$ & 18.867 & 0.002 & 19.095 & 0.044 & 18.654 & 0.021 \\
\hline SDSSJ232941.330+001107.755 & $23: 29: 41.325$ & $0: 11: 07.8$ & 18.323 & 0.002 & 18.394 & 0.028 & 18.187 & 0.021 \\
\hline SDSSJ235144.29+375542.6 & $23: 51: 44.293$ & $37: 55: 42.661$ & 18.272 & 0.002 & 18.417 & 0.014 & 18.056 & 0.016 \\
\hline
\end{tabular}


Table 4

Log of the Observations Collected with the Wide-Field Camera 3 on Board the Hubble Space Telescope during Cycles 20 and 22 (Proposal IDs 12967 and 13711, PI: A. Saha)

\begin{tabular}{|c|c|c|c|c|c|c|c|c|c|}
\hline Star & $\mathrm{PID}^{\mathrm{a}}$ & Image Name & $\begin{array}{c}\text { R.A. } \\
\text { (hh:mm:ss.s) }\end{array}$ & $\begin{array}{c}\text { Decl. } \\
\text { (dd:mm:ss.s) }\end{array}$ & Filter & $\begin{array}{c}\text { Exposure } \\
\text { Time } \\
\text { (s) }\end{array}$ & $\begin{array}{l}\text { Date Obs. } \\
\text { (YYYY/ } \\
\text { MM/DD) }\end{array}$ & $\begin{array}{l}\text { Time Obs. } \\
\text { (UT) }\end{array}$ & Aperture \\
\hline & & & & Cycle 20 & & & & & \\
\hline SDSSJ010322.19-002047.7 & 12967 & ibyn01wxq & 01:03:22.1 & $00: 20: 47.7$ & F336W & 160 & 2013 Sep 13 & $21: 46: 26$ & UVIS1-FIX \\
\hline SDSSJ010322.19-002047.7 & 12967 & ibyn01x0q & 01:03:22.1 & $00: 20: 47.7$ & F336W & 160 & 2013 Sep 13 & $21: 51: 25$ & UVIS1-FIX \\
\hline SDSSJ010322.19-002047.7 & 12967 & ibyn01xjq & $01: 03: 22.1$ & $00: 20: 47.7$ & $\mathrm{~F} 475 \mathrm{~W}$ & 160 & 2013 Sep 14 & 01:08:35 & UVIS1-FIX \\
\hline SDSSJ010322.19-002047.7 & 12967 & ibyn01xmq & 01:03:22.1 & $00: 20: 47.7$ & $\mathrm{~F} 475 \mathrm{~W}$ & 160 & 2013 Sep 14 & $01: 13: 33$ & UVIS1-FIX \\
\hline SDSSJ010322.19-002047.7 & 12967 & ibyn01wtq & 01:03:22.1 & $00: 20: 47.7$ & F625W & 350 & 2013 Sep 13 & $20: 30: 47$ & UVIS1-FIX \\
\hline SDSSJ010322.19-002047.7 & 12967 & ibyn $01 \times 8 q$ & 01:03:22.1 & $00: 20: 47.7$ & F625W & 355 & 2013 Sep 13 & $23: 36: 41$ & UVIS1-FIX \\
\hline SDSSJ010322.19-002047.7 & 12967 & ibyn01wqq & 01:03:22.1 & $00: 20: 47.7$ & F775W & 605 & 2013 Sep 13 & 20:18:08 & UVIS1-FIX \\
\hline SDSSJ010322.19-002047.7 & 12967 & ibyn $01 \times 5 q$ & 01:03:22.1 & $00: 20: 47.7$ & F775W & 680 & 2013 Sep 13 & $23: 22: 47$ & UVIS1-FIX \\
\hline
\end{tabular}

Note.

${ }^{\text {a }}$ Program ID.

(This table is available in its entirety in machine-readable form.)

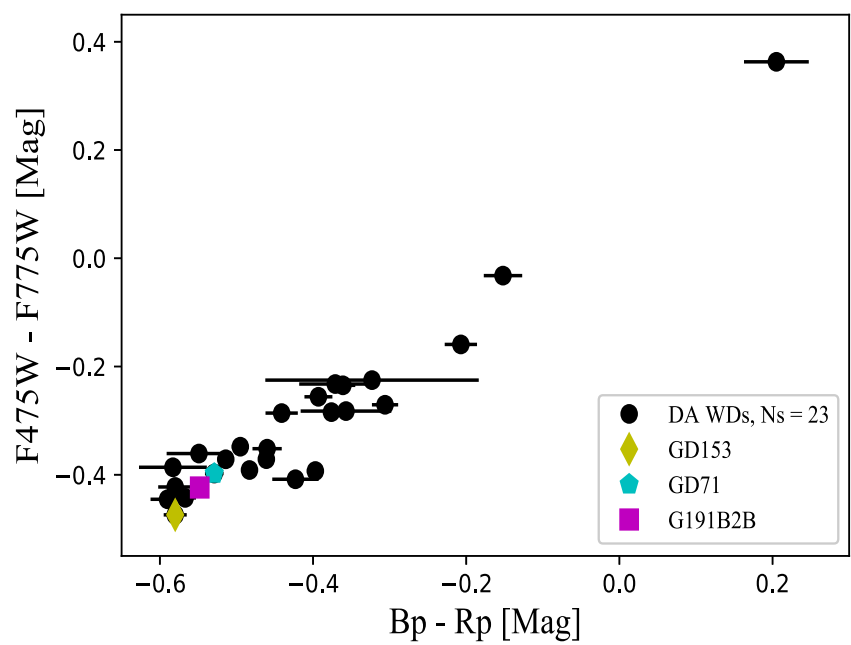

Figure 3. $B p-R p, F 475 W-F 775 W$ color-color diagram for the 23 selected DA white dwarfs (black dots) and the three HST primary standards: G191B2B (yellow diamond), GD153 (magenta square), and GD71 (cyan pentagon).

which is the cool DAWD included in our sample (as explained previously).

\section{Photometric Observations}

Photometric data discussed in this investigation were collected with the WFC3 UVIS and IR cameras on board the HST during Cycle 20 and 22 (proposals GO-12967 and GO13711, PI: Saha). Observations were taken in five filters in Cycle 20, namely F336W, F475W, F625W, F775W, F160W. In Cycle 22 the near-UV filter F275W was added to better characterize the line-of sight extinction and the reddening law toward the observed stars.

Nine of the candidate DAWDs are distributed along the celestial equator (hereinafter equatorial DAWDs) and were observed in both Cycle 20 and Cycle 22, while the other 14 DAWDs and the three $H S T$ primary CALSPEC standards were observed only in Cycle 22. Cycle 20 observations of the nine equatorial DAWDs were used in NA16 to demonstrate the effectiveness of our method to establish a network of spectrophotometric standards. We repeated the observations of these stars in Cycle 22 to improve the precision of the photometry. Moreover, photometry of the equatorial DAWDs will be used to determine the photometric offset between the two observing cycles. The $H S T$ primary CALSPEC WDs were observed in Cycle 22 to allow us to directly tie the photometry of the 23 DAWDs to the HST photometric system.

Exposure times for our observations range from 1 to $220 \mathrm{~s}$ for the F275W filter, 1 to $160 \mathrm{~s}$ for F336W, 1 to $160 \mathrm{~s}$ for F475W, 1 to $355 \mathrm{~s}$ for F625W, 1 to $680 \mathrm{~s}$ for F775W, and 3 to $499 \mathrm{~s}$ for F160W. Table 4 lists the log of the observations for Cycle 20 and 22.

Observations span a time interval of about $1 \mathrm{yr}$ for Cycle 20 (2012 November until 2013 September) and about $1.3 \mathrm{yr}$ for Cycle 22 (2014 September until 2016 January), with the HST primary CALSPEC WDs observed at the beginning and the end of Cycle 22 to track the change in sensitivity of the telescope and instrument system.

For the nine equatorial DAWDs, three dithered exposures in F336W and F475W, and two exposures for the other filters were collected in Cycle 20. The same targets were observed in Cycle 22 with a cosmic ray (CR) split of 3 exposures for F275W, and one exposure was added for the other filters. In summary, a total of three exposures for each of the WFC3 filters were collected for the nine equatorial DAWDs. At least three exposures per filter are needed to check consistency in the photometry at sub-percent precision: WFC3 images are affected by CRs and different detector artifacts, such as hot and bad pixels, blobs, ghosts. As an example, images in six filters for star SDSSJ232941.330+001107.755 are shown in Figure 4. If a star has discrepant measurements in two 

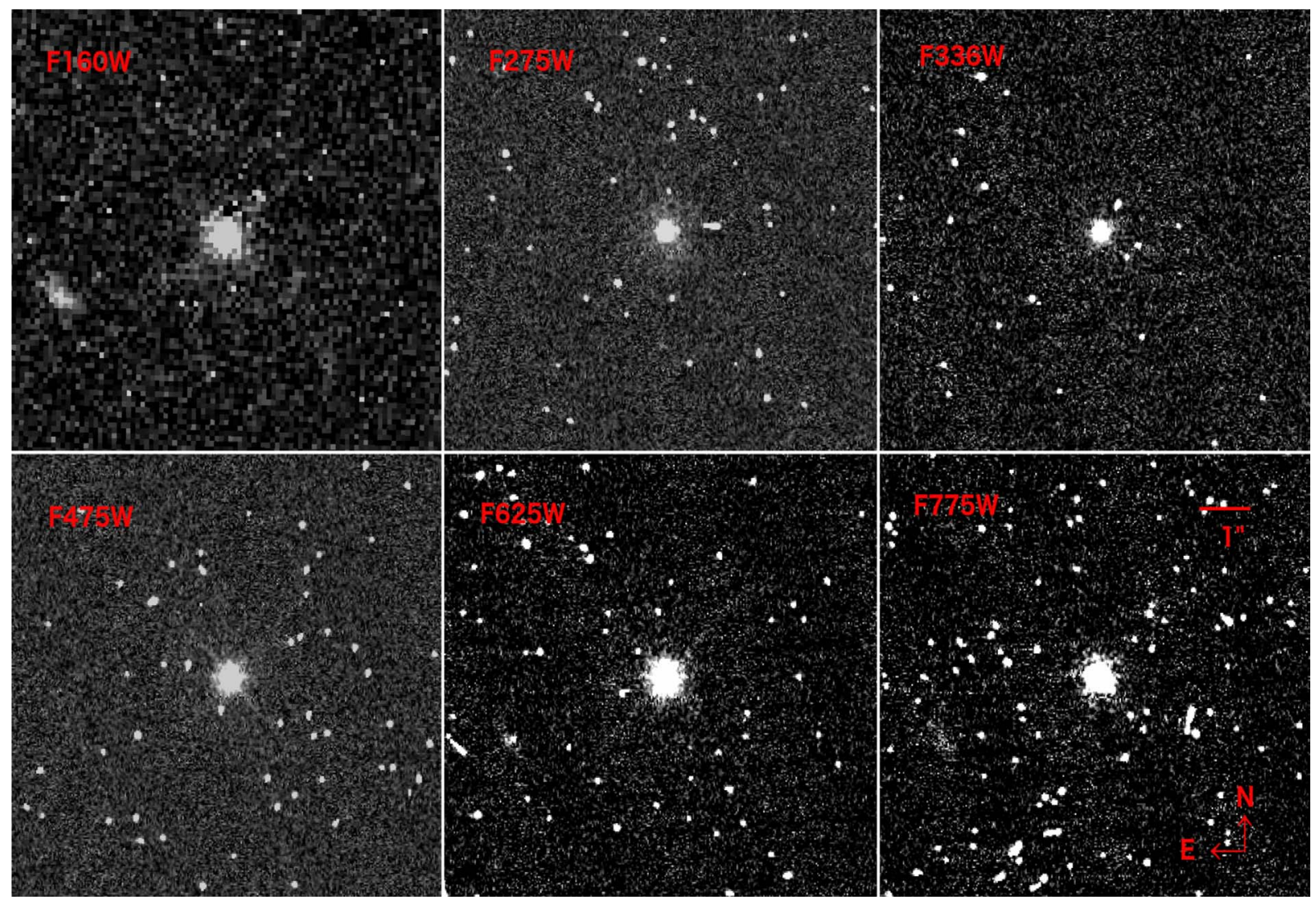

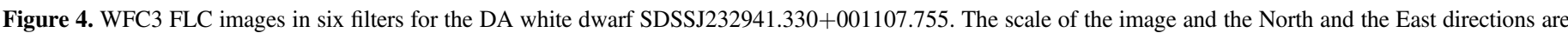
shown in the bottom right-hand panel. CRs and different detector artifacts, such as hot and bad pixels are visible in all images.

exposures, then the third image will allow us to identify the outlier measurement and discard the affected exposure.

The other 14 WDs were observed only in Cycle 22, by collecting five to seven dithered exposures for each filter. The HST primary CALSPEC standards were observed in Cycle 22 with six to eight exposures per visit, for a total of three visits (18 to 24 exposures) in all filters, spanning an average time interval of about $1 \mathrm{yr}$ and 3 months, from 2014 September/ November to 2015 November/2016 January. For more details on the observation strategy, please see the log in Table 4.

Parallel observations with the Advanced Camera for Surveys in the F475W and F775W filters were collected, including stars a few arcminutes away from the candidate spectrophotometric standards. The analysis of these images and an evaluation of the usefulness of the observed stars as supplementary standard stars will be presented in a forthcoming paper.

\subsection{Image Processing}

WFC3 UVIS images for Cycle 20 and Cycle 22 were processed with version 3.3 of the WFC 3 calibration pipeline (cal_wf3) that treats the two chips-UVIS1 and UVIS2individually (Deustua et al. 2017). The image photometry reference table (IMPHTTAB) that we used is $27 n 21066 i$ imp. fits and it writes PHOTFLAM values for a 10 pixel aperture (Deustua et al. 2016). A newer IMPHTTAB file was released in June 2017, which provides PHOTFLAM values for an infinite aperture, although it was not used to reduce our dataset. Cycle 20 images were collected by using the full UVIS1 aperture (UVIS1-FIX), with the target star placed in its center. Few pixel dithered exposures were collected to correct for detector artifacts and CRs (see the observation log in Table 4). The subarray UVIS2-C512C-SUB aperture was used for Cycle 22 observations, with the target star placed in its center and the exposures dithered by a few pixels (less than $\sim 20$ ). This allowed us to place the WDs closer to the read-out amplifier to mitigate the charge transfer inefficiency effects.

Starting from version 3.3, the WFC3 calibration pipeline scales UVIS2 fluxes to the UVIS1 chip by default. Therefore, we manually re-processed all the images with cal_wf3 by setting FLUXCORR = OMIT in the header to avoid the flux scaling and to keep the photometry on the UVIS2 detector system. Later on, the scale factor between Cycle 20 UVIS1 and Cycle 22 UVIS2 photometry will be estimated and applied to the measured magnitudes (see Section 4.4).

All of the WFC3-UVIS images were corrected for Charge Transfer Efficiency (CTE) by using the official WFC3 software $^{11}$ and the WFC3 Pixel Area Map (PAM) was applied $^{12}$ to correct for differences in the area of each pixel in the sky due to the geometric distortion of WFC3-UVIS.

WFC3 infrared (WFC3-IR) images were collected by using the full camera aperture (IR-FIX) and placing the targets at the center of the detector with every exposure dithered by 10-20 pixels. This strategy was used to avoid self-persistence. For the three primary WDs, observations were collected by using the IRSUB256-FIX and the IRSUB512-FIX subapertures to allow more exposures in the same orbit. Images were processed with the cal_wf3 calibration pipeline and the WFC3 PAM was applied.

\footnotetext{
11 http://www.stsci.edu/hst/wfc3/ins_performance/CTE

12 http://www.stsci.edu/hst/wfc3/pam/pixel_area_maps
} 


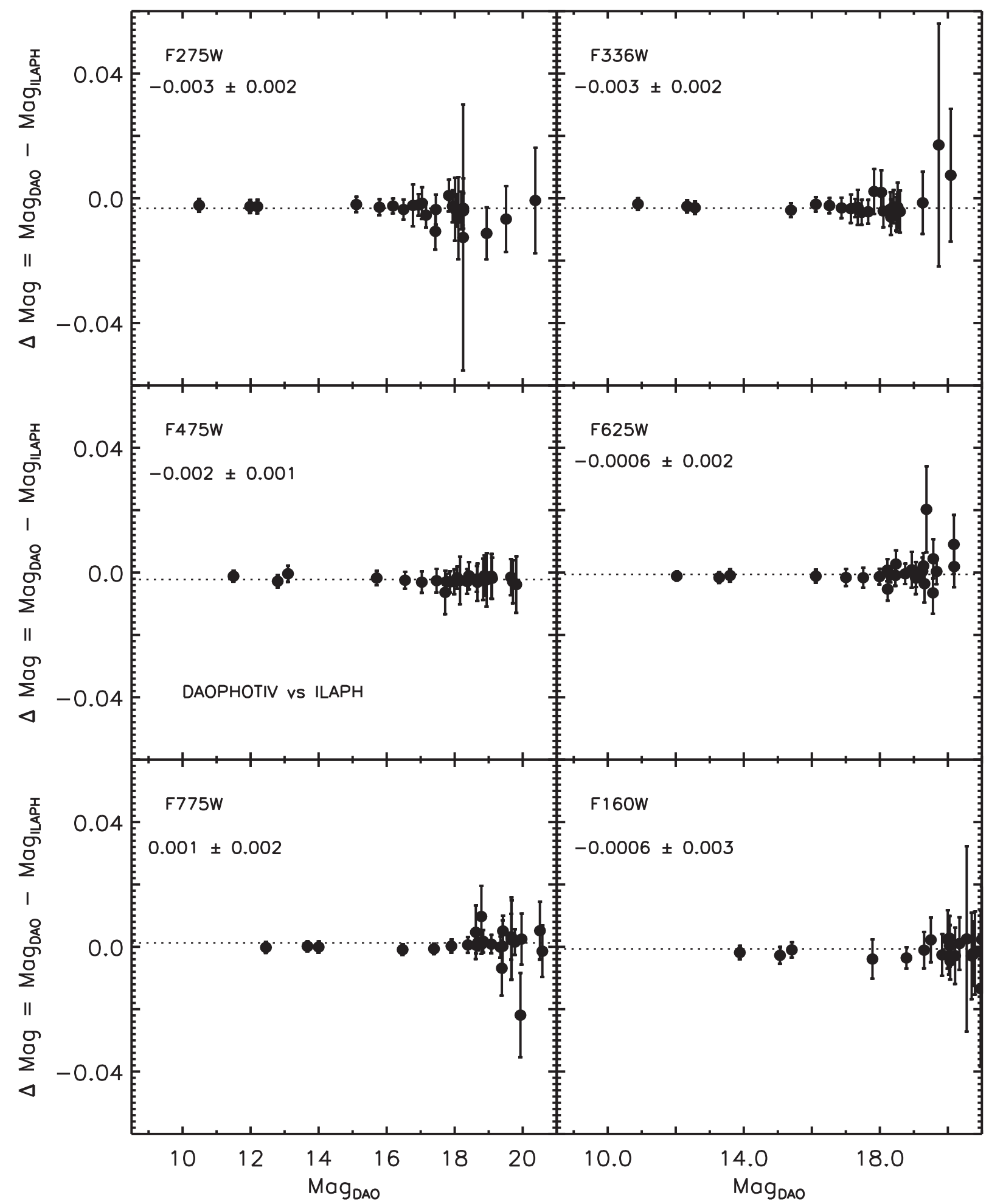

Figure 5. Comparison of DAOPHOT and ILAPH aperture photometry magnitudes, $\triangle \mathrm{Mag}=\mathrm{Mag}_{\mathrm{DAOPHOT}}-\mathrm{Mag}_{\mathrm{ILAPH}}$, in six filters as a function of DAOPHOT magnitude, for the 23 candidate spectrophotometric DAWDs and the $3 H S T$ primary CALSPEC standards. The mean magnitude differences with the relative dispersions are shown.

\section{Optimal Extraction of the White Dwarf Photometry}

Following a series of tests, we have discovered that the individual WFC3 images cannot be combined in the standard pipeline reduction and be expected to yield measurements with milli-mag level uncertainties. In particular, the anti-coincidence method of eliminating CR events can affect the cores of stars.
In reducing Cycle 20 WFC3 data, NA16 found that combining images with the drizzle algorithms (Drizzle $\mathrm{Pac}^{13}$ ) by using the pipeline default input parameters is not suitable for our purpose. Although drizzling eliminates CRs, it also introduces

$\overline{13}$ http://drizzlepac.stsci.edu/ 


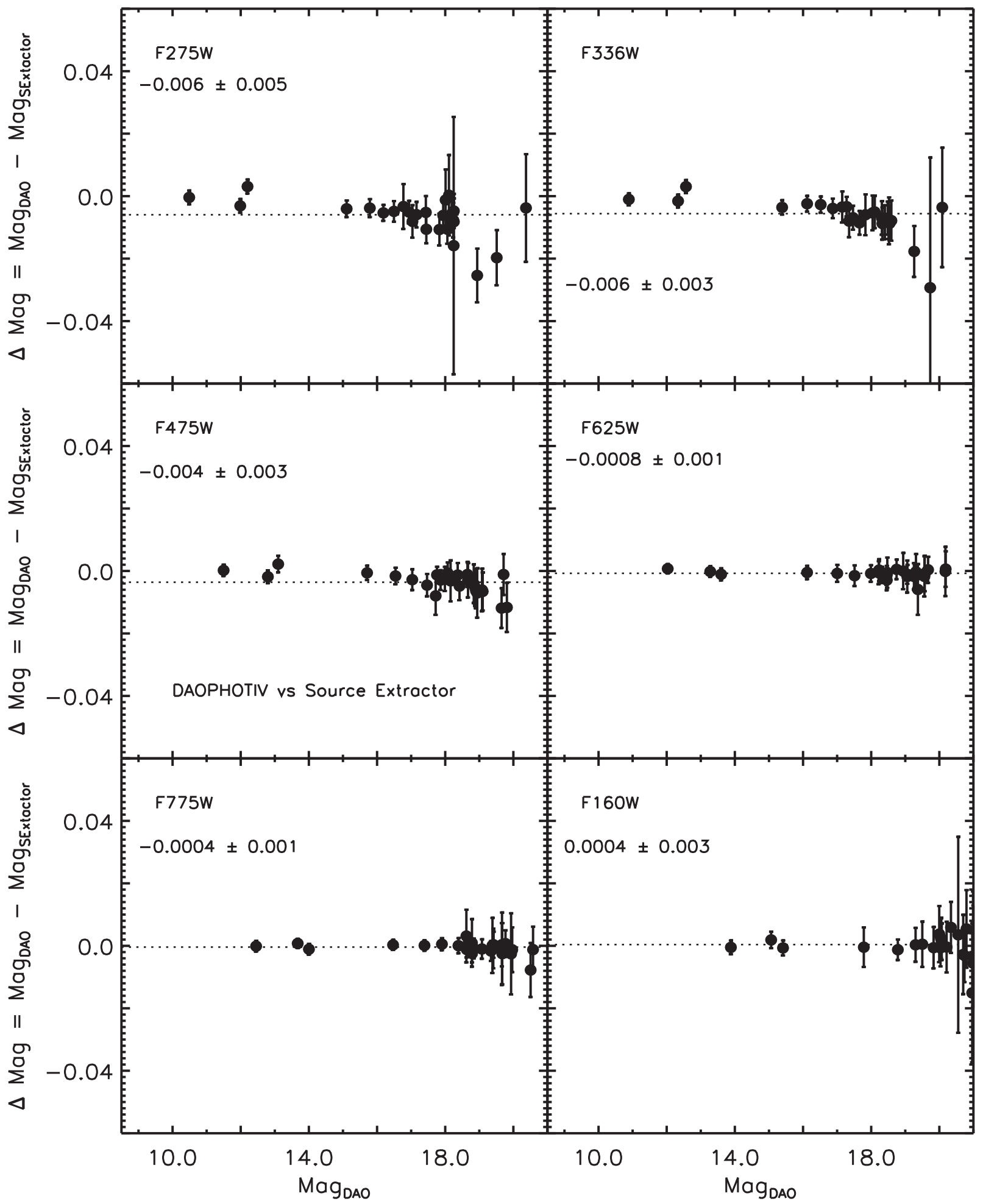

Figure 6. Same as Figure 5 but for DAOPHOT and SExtractor magnitudes, $\Delta \mathrm{Mag}=\mathrm{Mag}_{\mathrm{DAOPHOT}}-\mathrm{Mag}_{\mathrm{SExtractor}}$.

noise by over-correcting for differences in the cores of bright stars. Therefore, to avoid compromising the quality of the good images, where the target star is unaffected by a CR in its measuring aperture, we had to manually discard any image with a CR event over the measurement aperture on those few occasions it happened (3\%-4\% of the total number of images).

Our current reduction strategy involved performing photometry on the individual CTE-corrected images (FLC) for the
WFC3-UVIS detector, and on the FLT images for WFC3-IR, after having applied the PAM correction. We had to check all of the $\sim 800$ images for the presence of a CR event inside the star aperture radius. This was a rather tedious procedure but it allowed us to obtain a sub-percent accurate photometry for our set of standard stars.

We used three completely independent packages to perform aperture photometry on all of the images: (1) Source Extractor 


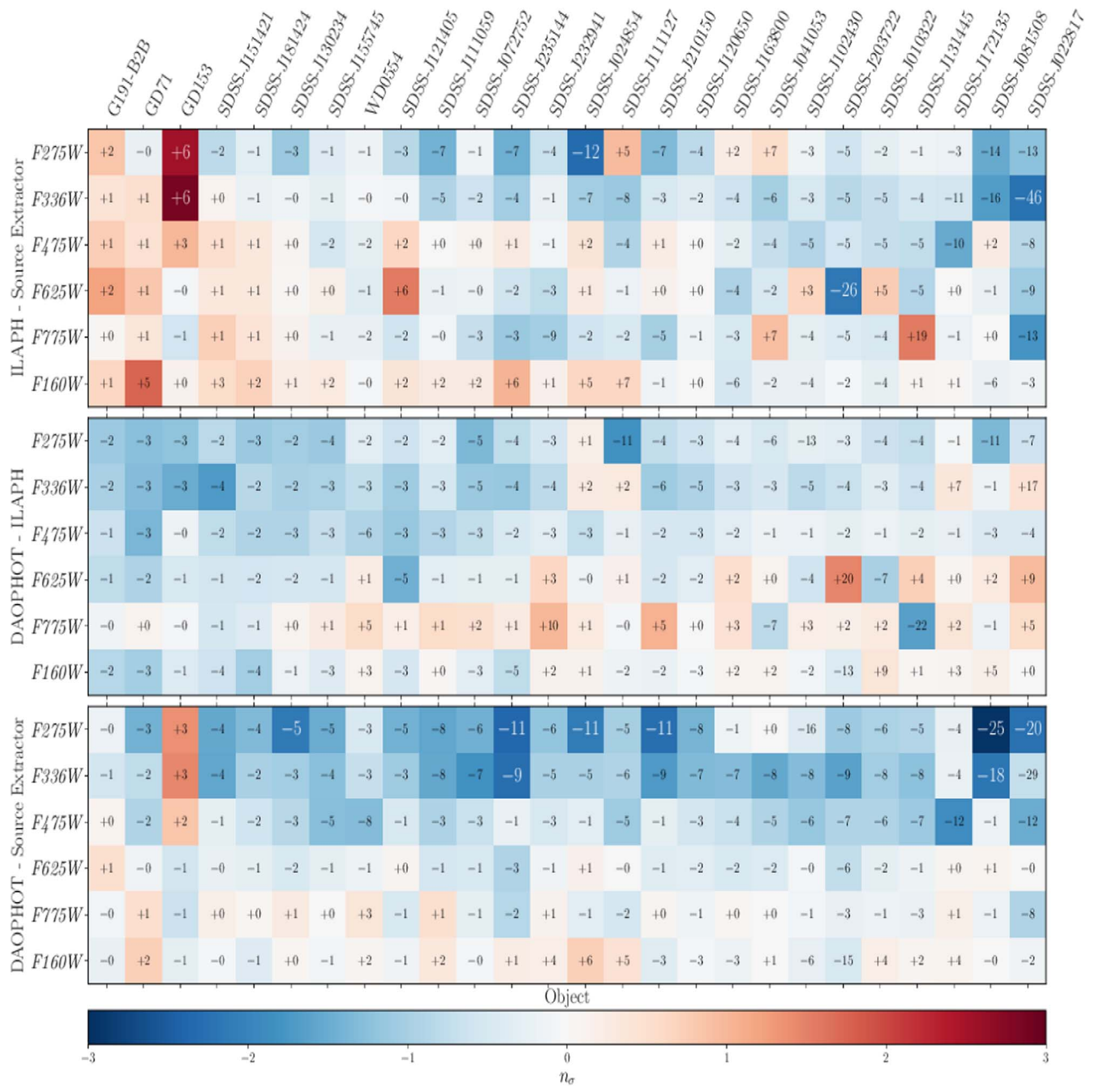

Figure 7. Comparison matrix between magnitudes measured with ILAPH, DAOPHOT, and Source Extractor for the 23 candidate standard DAWDs and the 3 HST primary CALSPEC stars in all the WFC3 observed filters. The color scale is labeled at the bottom of each panel and the name of the stars is marked at the top of the panels. Stars are sorted by $g$-band magnitude, starting with the brightest on the left. Filters are labeled on the left-hand side of each panel. The color of the box is bluer when the weighted magnitude difference between the two methods is negative (i.e., when the magnitude of the first labeled method is brighter compared to the magnitude of the second method), while it is red when the weighted magnitude difference is positive. See text for more details.

(Bertin \& Arnouts 1996, hereafter SExtractor); (2) DAOPHOTIV (Stetson 1987, hereafter DAOPHOT); and (3) ILAPH (an IDL based interactive program for aperture photometry and growth curve analysis written by A. Saha, customized for the data at hand).

Performing photometry by using different packages may generate systematic differences in the results. Our strategy will then enable us to track down possible systematic issues due to the data reduction method that we used. Moreover, it will allow us to determine realistic uncertainties, which is of paramount importance for our study because analysis downstream depends on these for weights.
After analyzing photometric growth curves for a sample of faint and bright WDs, we found the optimal aperture radius for the photometry to be 7.5 pixels for the WFC3-UVIS images and 5 pixels for the WFC3-IR images, i.e., $\sim 0$ !" 3 and $\sim 0$ "' 65 , respectively. The local sky background for each source was estimated in a rectangular region (SExtractor) and a circular (DAOPHOT, ILAPH) annulus around the aperture centered on the star. In the case of SExtractor, the box has a size of 20 pixels and the sky background in this region is estimated as a modified mode $(2.5 \times$ Median-1.5 $\times$ Mean $)$ after an iterative sigma-clipping rejection of the outlier pixels. DAOPHOT uses the mode $(3.0 \times$ Median $-2.0 \times$ Mean $)$ as the best sky 
estimator after an iterative sigma-clipping rejection of the outliers. However, in non-crowded stellar fields, if the mean sky value in the selected annulus is smaller than the median, then the mean of the sky value is used as best sky estimate. In this case, we used an annulus with radii $r_{\text {in }}=156$ and $r_{\text {out }}=165$ pixels around the target DAWDs. We selected these values since 156 pixels corresponds to $\sim 6^{\prime \prime}$ and it can be considered to infinity relative to the star's position.

ILAPH was configured to use the median sky value in the selected annulus around the stars as the best sky estimator. This is more robust than the mean because the latter is vulnerable to the presence of contaminating objects in the annular sky aperture. For UVIS, an aperture radius of 7.5 pixels and an annulus with radii $r_{\text {in }}=20$ and $r_{\text {out }}=30$ pixels was used for the sky. While this measurement procedure disregards light outside the respective apertures due to the extended skirt of the stellar point-spread function (PSF), it is asserted that the skirt affects all stars equally. In addition, as long as we measure the bright $H S T$ primary CALSPEC standards in exactly the same way as our target DAWDs, we are measuring instrumental magnitudes that all share a common ZP offset. For the IR images (F160W), ILAPH was used in the same way but with an aperture radius of 5 pixels and a sky aperture annulus from 14 to 21 pixels. The sky apertures were chosen with some experimentation: stability in the measured instrumental magnitude values from image to image for the same object was used as the criterion for selection. The program also looks at the pixel to pixel scatter within the annular sky aperture and it propagates that variance into the measurement error estimate. ILAPH was customized to use the actual fluctuation in the sky background and not just from the shot noise (Poisson statistics) of the adopted sky brightness in the calculation of photometric uncertainty. Subsequent analysis utilizes the uncertainties as weights, which makes it crucial to get this estimate to be as realistic as possible.

An accurate estimate of the sky background is fundamental for our analysis. In particular, a wrong estimate of the sky background has a greater effect on the faintest DAWDs and can introduce a systematic bias in the measurements. Figure 5 shows the comparison between the measurements in the six filters-F275W, F336W, F475W, F625W, F775W, and F160W-obtained with DAOPHOT and ILAPH $(\Delta$ Mag $=$ $\mathrm{Mag}_{\text {DAOPHOT }}-\mathrm{Mag}_{\mathrm{ILAPH}}$ ) for all the observed DAWDs as a function of the measured DAOPHOT magnitude. The single epoch magnitudes, corrected for the instrumental effects, including the sensitivity difference between Cycle 20 and Cycle 22, were averaged as described in Section 7.

The plot shows that measured aperture magnitudes with DAOPHOT and ILAPH are, within uncertainties, in very good agreement: with a biweight mean difference less than $0.003 \mathrm{mag}$ in all filters, and with a dispersion less than $0.003 \mathrm{mag}$ for all UVIS filters and less than $\sim 0.003 \mathrm{mag}$ for F160W. A couple of stars-SDSSJ102430.93-003207.0 and SDSSJ172135.97+294016.0 - show very large uncertainties, $\sim 0.02$ and $0.04 \mathrm{mag}$, respectively, in the $\mathrm{F} 275 \mathrm{~W}$ and the F336W filters. The first DAWD was already problematic in NA16 Cycle 20 measurements, and is a candidate variable (see Section 7.1). Star SDSSJ172135.97+294016.0. has no clear problems on the images, but an undetected faint CR falling on the aperture radius could be the culprit. However, this star is already excluded from our set of spectrophotometric standards due to its low effective temperature $\left(T_{\text {eff }} \sim 9000 \mathrm{~K}\right)$, so we will not investigate this issue further.

The same comparison is performed for all the measurements obtained with DAOPHOT and SExtractor in Figure 6, where $\Delta \mathrm{Mag}=\mathrm{Mag}_{\mathrm{DAOPHOT}}-\mathrm{Mag}_{\mathrm{SExtractor}}$. In this case, the measurements agree quite well within uncertainties but, on average, they have a larger dispersion, up to $0.005 \mathrm{mag}$ for F275W. Moreover, a slight trend with magnitude is present, with SExtractor magnitudes being fainter at fainter magnitudes.

To further investigate this issue, we produced a matrix comparison of the three data reduction methods in Figure 7. Each depicted box is color-scaled according to the weighted magnitude difference between two of the three methods, estimated as:

$$
\Delta \mathrm{Mag}=\left(\operatorname{Mag}_{\text {meth } 1}-\operatorname{Mag}_{\text {meth2 }}\right) / \sqrt{\operatorname{err}_{\text {meth } 1}^{2}+\operatorname{err}_{\text {meth2 }}^{2}}
$$

where $\mathrm{Mag}_{\text {methx }}$ and $\operatorname{err}_{\text {methx }}$ are the magnitudes and magnitude errors for each method, respectively.

Each box corresponds to a star measured in one of the six filters, sorted by magnitude (brightest on the left of the matrix), and the numeric text value is the magnitude difference, in millimag, between the measurements of the two methods. The color of the box is bluer when the weighted magnitude difference between the two methods is negative (i.e., when the magnitude of the first labeled method is brighter compared to the magnitude of the second method), while it is red when the weighted magnitude difference is positive. The text in the boxes is larger and white when the difference in magnitude between the two methods is larger than $2 \sigma$; i.e., significant compared to photometric errors.

The middle panel of the figure confirms that ILAPH and DAOPHOT provide very comparable results within the uncertainties, with no significant magnitude difference for all the measured stars in all filters. However, for fainter stars (right-hand section of the matrix), a very slight trend with color is present, in the sense that these stars are fainter in the redder filters (F625W, F775W, and F160W) when measured with DAOPHOT (redder boxes on the bottom right of the panel). However, this trend is well within the uncertainties of the measurements.

The top and the bottom panels of Figure 7 confirm that SExtractor magnitudes are systematically fainter compared to ILAPH and DAOPHOT magnitudes in all filters, as suggested by Figure 6 (bluer boxes in the panels). This difference has either a magnitude and a color effect: (1) magnitudes for brighter stars seems to agree between SExtractor and the other two methods, or to be brighter when measured with SExtractor (left part of the panels), while fainter stars are fainter when measured with SExtractor (right part); (2) the discrepancy is larger for the bluer filters (F275W and F336W, top part).

To understand this discrepancy, we compared the sky background values estimated with the three different methods. SExtractor local sky background shows systematically higher values when compared to DAOPHOT and ILAPH sky values. This difference in sky values is larger at fainter magnitudes, making SExtractor magnitudes fainter for fainter stars. However, the DAOPHOT and ILAPH sky values agree quite well within the uncertainties. 


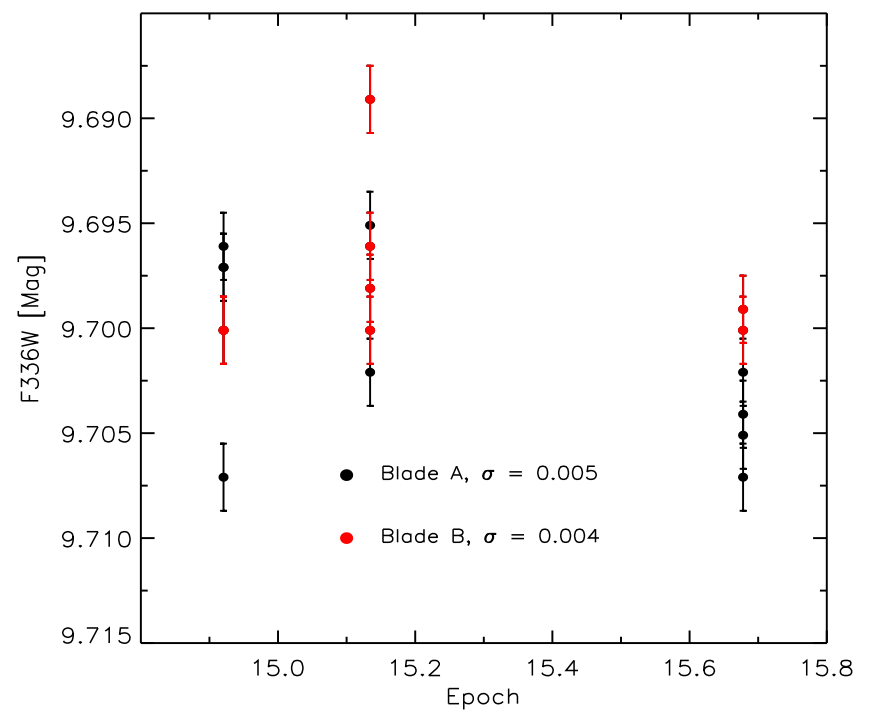

Figure 8. Aperture magnitudes in the F336W filter for star G191B2B measured with shutter blade A (black points) and B (red) plotted vs. the epoch. The error bars are labeled. The standard deviation of the measurements for the two blades is also shown.

\subsection{IR Photometry}

The WFC3-IR detector is affected by persistence; i.e., the residual signal of a large incident light level that can last on the images from minutes to days (Long et al. 2011, 2013; Gennaro et al. 2018). Some of our observations could have been scheduled after IR observations that cause persistence or bright objects in the images could have caused persistence in the same exposures. To verify this, we checked all of the images for the level of external (due to previous observations) or internal (due to objects in the same exposure) persistence by using the available WFC3 persistence tool. ${ }^{14}$ This search revealed that none of our observations was heavily affected by external or internal persistence, with the fraction of pixels with a residual signal larger than $0.01 \mathrm{e}^{-} \mathrm{s}^{-1}$ being less than $0.1 \%$. Note that the dark current signal for WFC3-IR is $0.04 \mathrm{e}^{-} \mathrm{s}^{-1}$. Three visits - one for star G191B2B, one for GD153 and one for SDSSJ181424.075+785403.048 - have a fraction of pixels with a residual signal larger than $0.01 \mathrm{e}^{-} \mathrm{s}^{-1}$ of $0.16 \%, 0.25 \%$ and $0.33 \%$, respectively, due to external persistence. However, the affected pixels do not overlap with the target DAWDs location, being more than 50 pixels away. Internal persistence is not an issue for all our observations. Brighter stars, such as the three primary WDs, could cause self-persistence on the IR images. To avoid this, we dithered each exposure by more than 10 pixels, as recommended by the WFC3 team.

Another issue affecting IR observations is the count-rate nonlinearity (CRNL); i.e., the nonlinearity of the detected counts with the total incident flux on the camera. This effect may be relevant for our observations because the target DAWDs cover a range of more than $10 \mathrm{mag}$. The CRNL was characterized for the WFC3-IR camera by Riess $(2010,2011)$, Riess \& Petro (2010) and who measured $0.010 \pm 0.0025 \mathrm{mag}$ per dex for the F160W filter. The net effect of CRNL is that photometry of very faint stars-i.e., background dominatedappears fainter (Riess \& Petro 2010). An accurate characterization of the CRNL for the program IR photometry, based on the observations and on models, will be provided in NA19.

\footnotetext{
${ }^{14}$ https://archive.stsci.edu/prepds/persist/search.php
}

Therefore, we do not apply any CRNL corrections on the photometry presented in this paper.

\subsection{Shutter Shading}

The accuracy of the measured magnitudes on WFC3-UVIS could be affected by the shutter shading effect. For the brightest stars in our sample, we used very short exposures times $(t<2 \mathrm{~s})$. For these short times, shutter vibration can affect the actual duration of the exposures, leading to fainter measured magnitudes on the image. This effect was studied in detail by the WFC3 team and discussed in different Instrument Science Reports (Hilbert 2009; Sabbi 2009; Sahu et al. 2014, 2015). Shutter vibrations in short exposures also results in a broadening of the observed PSF. When observing using shutter blade $\mathrm{B}$, the Full-Width-Half-Maximum of the stellar images is systematically larger than when using blade A. The larger shutter vibrations when using blade $\mathrm{B}$ can introduce a flux measurement uncertainty up to $\sim 2 \%$ for photometry performed with aperture radii smaller than 5 pixels (Sabbi 2009).

For our WFC3-UVIS data, we used an aperture radius of 7.5 pixels and so shutter shading should not affect images collected by using blade B. However, we checked for the presence of this effect on the images of the brightest of the primary CALSPEC standards-G191B2B. The selected F336W images were collected in a sequence of $1.0 \mathrm{~s}$ exposures alternating the two shutter blades (ABAB...). Figure 8 shows instrumental aperture magnitudes in the $\mathrm{F} 336 \mathrm{~W}$ filter for G191B2B measured with shutter blade A (black points) and B (red) plotted versus the observing epoch. The standard deviation of the measurements is 0.005 and 0.004 mag for blade $\mathrm{A}$ and $\mathrm{B}$, respectively. The plot shows that there is not significant difference between magnitudes measured on images collected by using blade A or B. We performed the same experiment for G191B2B images collected with the other UVIS filters and obtained similar results.

The exposures times for the other DAWDs are longer than for G191B2B (see the observing log in Table 4), so we did not verify for shutter blade effects in all the other observations and we can safely assume that none of the exposures is affected.

\subsection{Testing Photometry for Crowding Effects}

Another factor that could affect the accuracy of measured magnitudes is the presence of unseen neighbor stars in the DAWD aperture radius. To test this hypothesis, we performed some artificial star (AS) tests simulating stars of different brightness with centroids from 0 to 5 pixels away from the DAWD. We simulated stars from 3 to 7 mag fainter than the DAWDs. The results of these simulations show that AS more than 6 mag fainter than the target DAWD falling in the 7.5 pixel aperture radius do not affect the measured magnitudes. Meanwhile, brighter neighbor stars falling inside the aperture radius could affect the photometry of the target DAWD by adding $\sim 1 \%$ of noise to the measurement.

However, our candidate standard DAWDs are in sparsely populated stellar fields and the observed WFC3 sub-array field of view (FoV) is $\sim 20^{\prime \prime}$. We checked the PS catalog to look for the presence of other stars in the observed FoV and found only the target DAWDs or a maximum of other two objects (well outside the aperture radius) down to the PS detection limit ( $g \sim 23 \mathrm{mag}$ ), so more than 5 mag fainter than our targets. We 


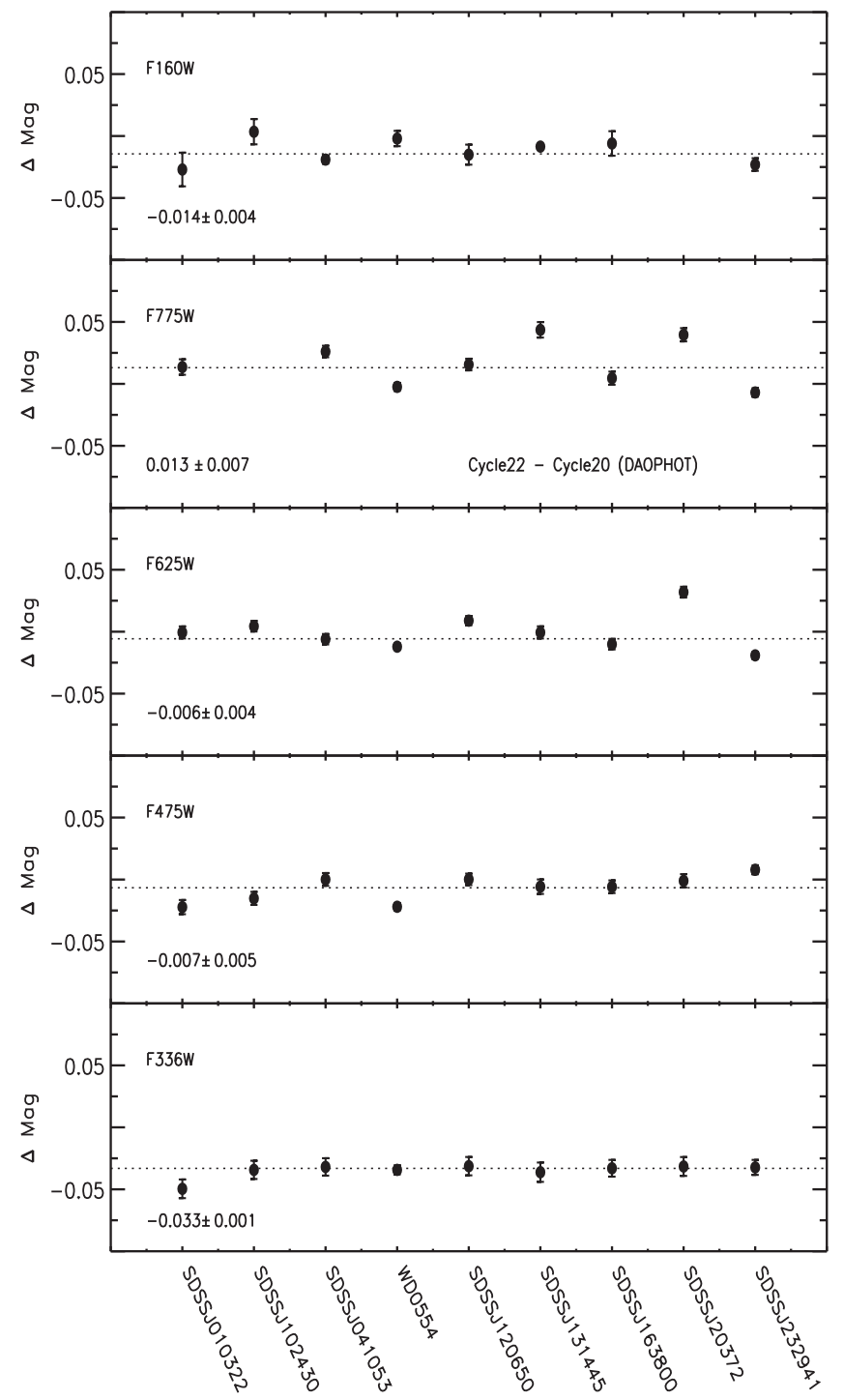

Figure 9. Comparison of Cycle 20 and Cycle 22 average magnitudes measured with DAOPHOT in six filters, namely F275W, F336W, F475W, F625, F775W, and $\mathrm{F} 160 \mathrm{~W}$ for nine equatorial WDs. The weighted mean magnitude differences between the two observing cycles with the relative errors are also labeled.

can then safely assume that the photometry of the DAWDs is not affected by contamination of unseen neighbor stars.

\subsection{Magnitude Offset between the HST Observing Cycles}

Images in the five filters-F336W, F475W, F625W, F775W, and F160W-were collected in Cycle 20 and Cycle 22 for the nine equatorial WDs. For this subset of targets, we then have two sets of measurements. Because the primary CALSPEC WDs, which anchor our photometry to the HST system, were only observed in Cycle 22, we need to estimate the magnitude offset between the two cycles and calibrate Cycle 20 measurements to Cycle 22. Cycle 20 observations were performed by using the full UVIS1 aperture, while Cycle 22 exposures were collected with a UVIS2 sub-array. The magnitude offset needs to take into account the difference due to observing with two different WFC3 detectors and all the effects due to observations taken more than $2 \mathrm{yr}$ apart.
Figure 9 shows the comparison between Cycle 20 and Cycle 22 magnitudes in the five filters for the nine equatorial WDs. Star SDSSJ102430.93-003207.0 has very discrepant measurements in the F775W filter ( $\Delta$ Mag $\sim-0.21$ ); NA16 claim that this WD might be variable. The same applies to star SDSSJ203722.169-051302.964, where the F160W measurements are in strong disagreement between the two observing cycles $(\Delta$ Mag $\sim 0.27$ ). The spectrum of this WD shows emission features in the core of the Balmer absorption lines, which indicates the presence of a low-mass companion star (see Section 6 for more details). Therefore, both stars were removed from the sample to estimate the magnitude offset.

The offsets are between $\sim 0.005$ and $\sim 0.03$ mag, depending on the filter, with an average dispersion of 0.005 mag. Columns 8 and 9 of Table 6 list the magnitude offsets and their uncertainties between the two HST observing cycles in the different filters.

After having applied these magnitude offsets, we estimated the weighted mean instrumental magnitudes for all the 23 observed DAWDs based on the photometry of the two cycles.

\section{Photometric Stability of the Candidate Standard DAWDs}

To assess the 23 candidates as stable standards, we monitored them by collecting time-series data with the Las Cumbres Observatory (LCO) network of telescopes (proposals LCO2016B-007, LCO2017AB-002, PI: Matheson).

WDs can vary for several reasons, depending on their effective temperature, atmosphere abundance and presence of magnetic activity or of an unseen faint companion star.

Hydrogen-rich atmosphere WDs might present gravity-mode pulsations around $T_{\text {eff }} \sim 12,000 \mathrm{~K}$ (Fontaine \& Brassard 2008, ZZ Ceti pulsators). Our DAWDs were selected to have temperatures $\left(T_{\text {eff }} \gtrsim 20,000 \mathrm{~K}\right)$ outside the ZZ Ceti instability strip, so we do not expect them to be pulsators (note that SDSS$\mathrm{J} 172135.97+294016.0$ will be removed from the network of standards since it has $T_{\text {eff }}=9261 \mathrm{~K}$ ). Strong magnetic fields can also cause flux variations in WDs with a timescale from hours to days. These variations can be due to magnetically confined spots of higher opacity modulating the stellar flux via stellar rotation (Dupuis et al. 2000; Holberg \& Howell 2011). Alternately, magnetic variations can be due to to spots in the convective atmosphere (Brinkworth et al. 2004, 2013). However, the 23 candidate standard DAWDs have effective temperatures above $\sim 20,000 \mathrm{~K}$, and their atmosphere are fully radiative, so they should not vary due to the presence of spots. Moreover, their spectra did not show Zeeman splitting of the Balmer lines indicative of the presence of a strong magnetic field (see Section 6). However, the selected DAWDs could still vary due to the presence of an unseen faint companion star, or to unknown factors, and we need to characterize the amount of flux variation, if present, before setting these stars as spectrophotometric standards. A recent study by Hermes et al. (2017), based on precise Kepler time-series photometry, showed that $\sim 97 \%$ of apparently isolated WDs are stable or show less than $1 \%$ flux variations and can still be used as spectrophotometric standards. Hermes et al.'s sample included mostly DAWDs and also included several helium- or carbon-dominated atmosphere WDs, with temperatures hotter than $\sim 8000 \mathrm{~K}$.

On the basis of the criteria used to select the 23 DAWDs and previous studies, we do not expect a large fraction of our candidate spectrophotometric standards to vary. However, 


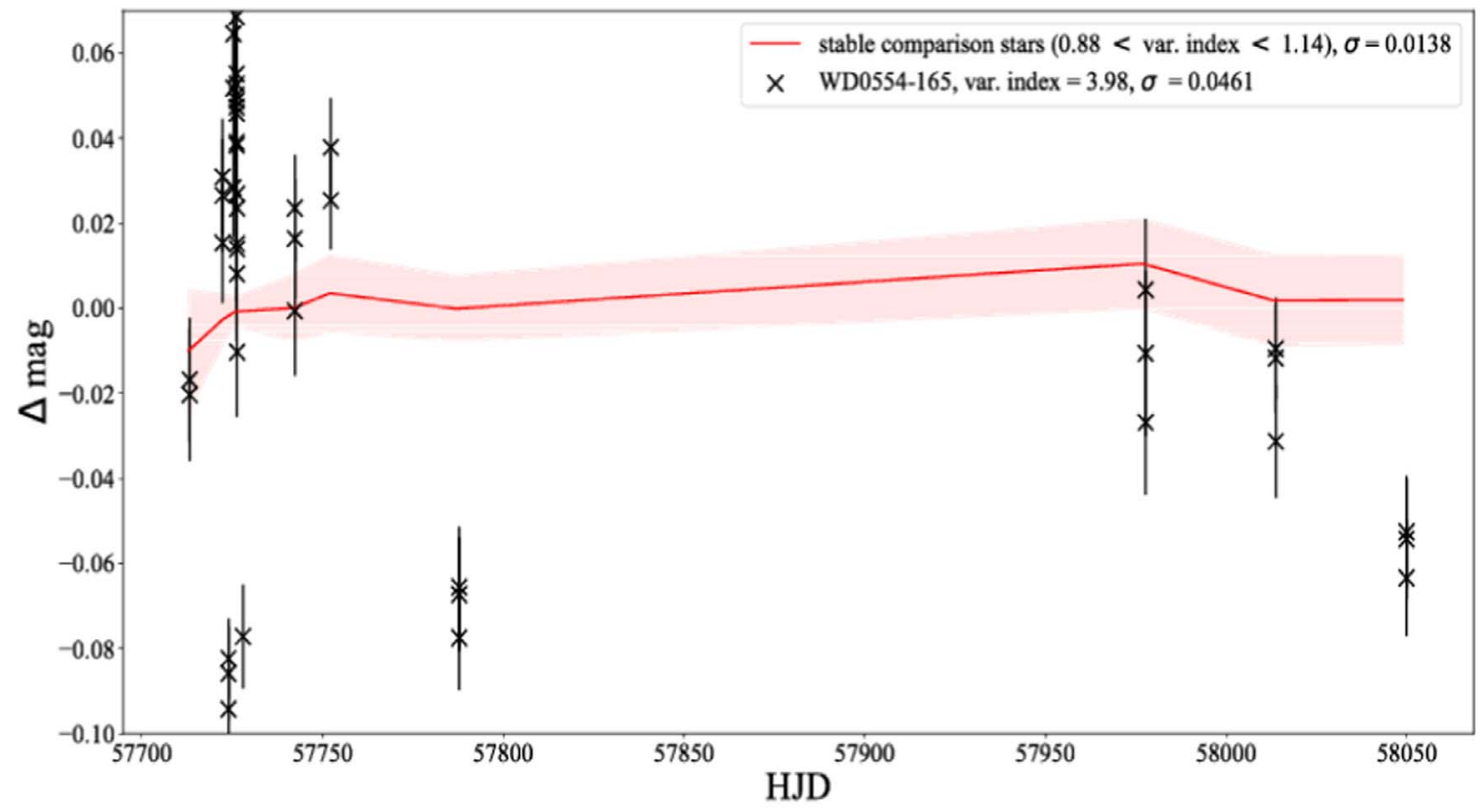

Figure 10. Single epoch minus the mean instrumental magnitude measurements for WD0554-165 as a function of observing epoch (black crosses). Averaged and binned relative magnitudes for a set of stable stars of comparable instrumental magnitude in the same field of view are overplotted as a red shaded area. The variability index of the selected stars and the measurement dispersions are listed. Error bars are shown.

these 23 DAWDs have not yet been subject to a consistent and well-defined observational campaign to demonstrate a lack of variability at a wide range of timescales. WFC3 observations are obtained within a short time frame for each target, which makes them unsuitable as tests of variation. In addition, prior ground-based surveys (SDSS, PanSTARRS) do not have the necessary temporal coverage and GAIA does not yet provide variability constraints on these stars.

\subsection{Time-series Photometry}

LCO observations consist of a sequence of geometrically spaced exposures in the Sloan $g$ filter, ranging from minutes to month-long timescales. A minimum of 20 exposures for each target were collected, spread over 2-3 months at different time intervals, for a total of about 800 images.

PSF photometry was performed with DAOPHOTIV/ALLSTAR (Stetson 1987) and ALLFRAME (Stetson 1994). The average FWHM for each frame was measured by using Source Extractor to exclude observations affected by poor observing conditions or bad focus, and these handful of images were excluded from the analysis. All the exposures for each target were flux scaled to the best image, defined by the frame with the smallest average FWHM, which was used as a reference image. Light curves were then produced for each of the 23 targets.

To select candidate variables we used the Welch \& Stetson (1993) variability index $W$ :

$$
W=\sqrt{\frac{1}{n(n-1)}} \sum_{i=1}^{n} \frac{m_{i}-\bar{m}}{\sigma_{i}}
$$

where $m_{i}$ are the individual measurements and $\bar{m}$ is the mean weighted magnitude of each identified object, and $n$ is the total number of frames. The Welch-Stetson variability index was calculated for all of the stars (from $\sim 500$ to 1000) in the FoV. A sample of stable comparison stars was selected for each of the 23 DAWD observation. This group of stable stars has a detection in every frame and a variability index, var index, $\leqslant 1.2$, the sharpness of the PSF in the range $-0.5<$ sharpness $<0.5$, to exclude extended objects and CRs, and a proximity in magnitude to the target WD within $\sim 0.2 \mathrm{mag}$.

An absolute calibration of the photometry is not performed because our goal is to demonstrate the lack of variability of the candidate standard DAWDs. However, we need to take into account spurious flux variations due to instrumental and atmospheric effects (observations are performed with different telescopes and detectors and from different sites in different conditions). The light curves of the selected stable stars are then compared to the light curves of the WDs in the same field. The variation around the mean of the stable star magnitudes was averaged and the average $1 \sigma$ dispersion was estimated. This dispersion is used as a variability threshold for the systematic observational and instrumental effects.

Figure 10 shows the single epoch minus the weighted mean instrumental magnitude as a function of the Heliocentric Julian Date (HJD) for WD0554-165 (black crosses). The averaged and binned relative magnitudes for a set of stable stars of comparable instrumental magnitude in the same FoV are also plotted as a red shaded area. The selected comparison stars have a variability index less than 1.2 while the WD0554-165 has a variability index of 3.98. WD0554-165 shows clear signs of variability, with variations of almost $0.2 \mathrm{mag}$ and a measurement $1 \sigma$ dispersion of $\sim 0.05 \mathrm{mag}$, compared to the stable star dispersion of $\sigma \sim 0.01 \mathrm{mag}$. Figure 11 shows the same plot but for a stable DAWD, SDSSJ235144.29 +375542.6 , whose variability index is $\sim 1$ and the dispersion of the measurement is $\sim 0.015 \mathrm{mag}$, which is smaller than the measurement dispersions for the stable stars, $\sigma \sim 0.018 \mathrm{mag}$.

The light curve for SDSSJ20372.169-051302.964, which is a candidate binary system from spectroscopic data, shows variability with a dispersion of the measurements of $\sigma \sim$ $0.04 \mathrm{mag}$, which is a factor of 4 larger than the comparison star 


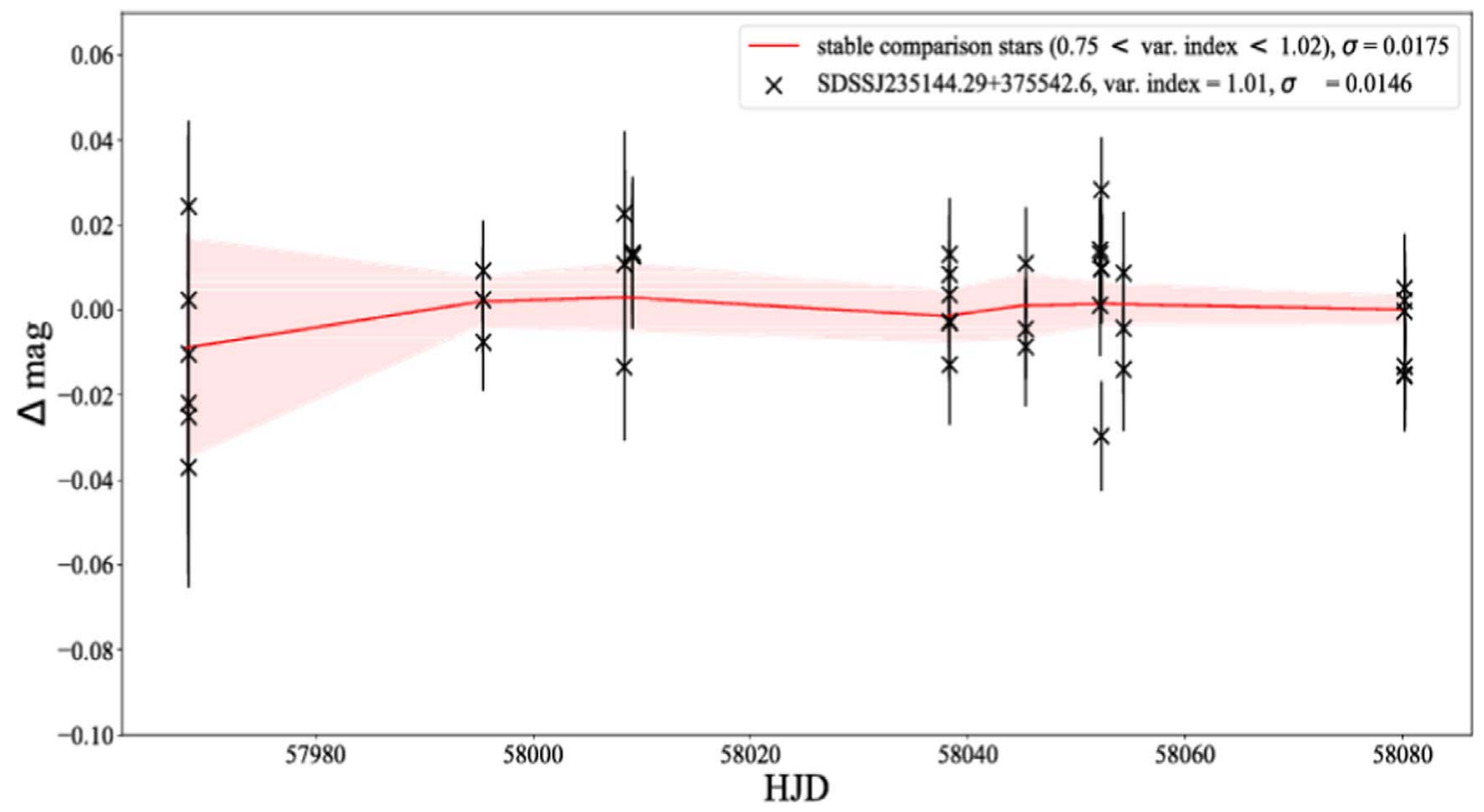

Figure 11. Same as Figure 10 but for star SDSSJ235144.29+375542.6.

measurement dispersion, $\sigma \sim 0.01 \mathrm{mag}$, thus confirming its binary nature.

Although the other two stars in the sampleSDSSJ010322.10-002047.7 and SDSSJ102430.93-003207.0 - show hints of variability, more and deeper exposures are needed to confirm these preliminary results.

Stars SDSSJ20372.169-051302.964 and WD0554-165 will be excluded from our network of spectrophotometric standard DAWDs due to their variable nature.

A more detailed analysis of the LCO photometry and the DAWD light curves will be presented in a forthcoming paper. We also plan to follow-up the candidate variable DAWDs with more observations from a larger ground-based telescope to understand the origin of their variability.

\section{Spectroscopic Observations}

The spectra of the DAWDs are used to determine $T_{\text {eff }}$ and $\log g$. These values are derived from the shape of the H I Balmer line profiles from $\mathrm{H} \beta$ to $\mathrm{H} \zeta$. We flux-calibrated the spectra to facilitate the analysis of the Balmer lines but the overall shape of the spectrum will retain uncertainties introduced by the flux calibration process and by the inherent uncertainty in the standard stars used. We emphasize that the spectral shapes are not critical to the ultimate analysis of these DAWDs as spectrophotometric standards. The deviation of the calibrated spectral shape from a model spectrum is treated as a nuisance parameter in the fitting process, thus minimizing the calibration error improves the uncertainties in the end result. Nonetheless, the values of $T_{\text {eff }}$ and $\log g$ from the Balmer lines in concert with the photometry provide the ultimate calibration of these stars.

We used two different facilities to obtain spectra of our standard star candidates. As part of the HST photometry proposal, we were awarded Gemini time. This amounted to $43 \mathrm{hr}$ from Cycle 20 (split over Gemini semesters 2013A and 2013B) and $28.1 \mathrm{hr}$ from Cycle 22 (split over Gemini semesters 2015A and 2015B). For most of this time, we used Gemini South, but we also used Gemini North for the northern targets. At each site, we used the Gemini Multi-Object Spectrograph (GMOS, Hook et al. 2004 ) in queue mode with a long slit to obtain the spectra. For the 2013A and 2013B semesters, we used the 1."5 slit; while for $2015 \mathrm{~A}$ and $2015 \mathrm{~B}$, we used the $1{ }^{\prime \prime} 0$ slit. The three GMOS detectors are not contiguous, so we used two different grating tilts to fill in the inter-chip gaps. The final spectra are continuous from 3500 to $6360 \AA$ with a dispersion of $0.92 \AA /$ pixel. The resolution of the spectra is a function of the seeing at the time of observation given the relatively wide slit and the generally good seeing conditions at the Gemini sites. Determining the resolution is an element of the data analysis process that will be described in a later paper.

We found that the Gemini data were generally not of sufficiently high quality for our purpose. The throughput of the GMOS system in the blue is poor. In addition, standard stars and other calibrations were frequently not obtained in conjunction with the spectra of the white dwarfs. Finally, despite our request, the observations were typically not obtained at the parallactic angle (Filippenko 1982) so slit losses resulting from atmospheric dispersion resulted in compromised shapes for the SEDs of the stars. Because of these issues, we instituted a program at the MMT Observatory to obtain alternate spectra of our DAWDs.

At the MMT, we used the Blue Channel spectrograph (Schmidt et al. 1989) with the 300 line $\mathrm{mm}^{-1}$ grating. We had a total of four successful observing nights spread over three epochs. For most of the observations, we used the 1 ". 0 slit, but we used the 1 ". 25 slit for one epoch. The wavelength coverage runs from 3400 to $8400 \AA$ with a dispersion of $1.95 \AA$ /pixel. All observations were obtained at the parallactic angle and standard stars were observed on the same night. As with the GMOS data, the resolution of the spectra depends on the seeing at the time of observation.

Details of the observations with both facilities are presented in Table 5. We used standard IRAF $^{15}$ routines to process the

\footnotetext{
15 IRAF is distributed by the National Optical Astronomy Observatory, which is operated by AURA under cooperative agreement with the NSF.
} 
Table 5

Log of the Spectroscopic Observations

\begin{tabular}{|c|c|c|c|c|c|c|c|c|c|}
\hline Star & UT Date & Tel. $^{\mathrm{a}}$ & $\begin{array}{c}\text { Range } \\
(\AA)\end{array}$ & 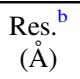 & $\begin{array}{l}\text { P.A. }^{c} \\
\left({ }^{\circ}\right)\end{array}$ & Airmass & Flux Std. ${ }^{\mathrm{d}}$ & $\begin{array}{l}\text { Slit } \\
(")\end{array}$ & $\begin{array}{r}\text { Exposure } \\
\text { (s) }\end{array}$ \\
\hline G191B2B & 2015 Jan 24 & MMTO & $3400-8400$ & 8 & -111.8 & 1.2 & Feige34 & 1.0 & 8 \\
\hline GD153 & 2015 May 18 & MMTO & $3400-8400$ & 8 & -52.9 & 1.0 & $\mathrm{BD}+284211$ & 1.0 & 15 \\
\hline GD71 & 2015 Jan 24 & MMTO & $3400-8400$ & 8 & 12.8 & 1.0 & Feige34 & 1.0 & 10 \\
\hline SDSSJ010322.19-002047.7 & 2013 Nov 29 & GEMINI-S & $3500-6360$ & 10 & 180.0 & 1.2 & GD71 & 1.5 & $6 \times 1500$ \\
\hline SDSSJ010322.19-002047.7 & 2015 Oct 11 & MMTO & $3400-8400$ & 8 & 13.5 & 1.2 & $\mathrm{BD}+284211$ & 1.25 & $2 \times 1200$ \\
\hline SDSSJ022817.16-082716.4 & 2013 Oct $23^{\mathrm{e}}$ & GEMINI-S & $3500-6360$ & 10 & 0.0 & 1.2 & GD71 & 1.5 & $7 \times 1500$ \\
\hline SDSSJ024854.96+334548.3 & 2015 Oct $08^{\mathrm{e}}$ & GEMINI-N & $3520-6360$ & 7 & 232.0 & 1.1 & $\mathrm{BD}+284211 / \mathrm{G} 191 \mathrm{~B} 2 \mathrm{~B}$ & 1.0 & $8 \times 999$ \\
\hline SDSSJ024854.96+334548.3 & 2015 Oct 11.5 & MMTO & $3400-8400$ & 8 & 92.4 & 1.1 & $\mathrm{BD}+284211$ & 1.25 & $5 \times 1200$ \\
\hline SDSSJ041053.632-063027.580 & $2013 \mathrm{Dec}_{04}^{\mathrm{e}}$ & GEMINI-S & $3500-6360$ & 10 & 180.0 & 1.2 & GD71 & 1.5 & $6 \times 1500$ \\
\hline SDSSJ041053.632-063027.580 & 2015 Jan 24 & MMTO & $3400-8400$ & 8 & 12.8 & 1.3 & Feige34 & 1.0 & $3 \times 900$ \\
\hline WD0554-165 & 2015 Jan 24 & MMTO & $3400-8400$ & 8 & 12.8 & 1.5 & Feige34 & 1.0 & $3 \times 900$ \\
\hline SDSSJ072752.76+321416.1 & 2015 Jan 24 & МMTO & $3400-8400$ & 8 & -93.5 & 1.0 & Feige34 & 1.0 & $3 \times 900$ \\
\hline SDSSJ081508.78+073145.7 & $2013 \mathrm{Jul} 07^{\mathrm{e}}$ & GEMINI-S & $3500-6360$ & 12 & 0.0 & 1.3 & GD71/Feige110 & 1.5 & $6 \times 1500$ \\
\hline SDSSJ081508.78+073145.7 & 2015 Jan 24 & MMTO & $3400-8400$ & 8 & 9.3 & 1.1 & Feige34 & 1.0 & $4 \times 900$ \\
\hline SDSSJ102430.93-003207.0 & 2013 Feb 15 & GEMINI-S & $3500-6360$ & 12 & 0.0 & 1.3 & Feige110 & 1.5 & $6 \times 1500$ \\
\hline SDSSJ111059.43-170954.1 & 2015 Jan 24 & MMTO & $3400-8400$ & 8 & -5.2 & 1.5 & Feige34 & 1.0 & $3 \times 900$ \\
\hline SDSSJ111059.43-170954.1 & 2015 May 18 & GEMINI-S & $3500-6500$ & 8 & 0.0 & 1.2 & Feige67 & 1.0 & $8 \times 700$ \\
\hline SDSSJ111127.30+395628.0 & 2015 Jan 24 & MMTO & $3400-8400$ & 8 & -111.6 & 1.0 & Feige34 & 1.0 & $3 \times 900$ \\
\hline SDSSJ111127.30+395628.0 & 2015 May 18 & MMTO & $3400-8400$ & 8 & 130.0 & 1.0 & $\mathrm{BD}+284211$ & 1.0 & $2 \times 900$ \\
\hline SDSSJ120650.504+020143.810 & 2013 Mar 10 & GEMINI-S & $3500-6360$ & 10 & 35.0 & 1.2 & Feige110 & 1.5 & $6 \times 1500$ \\
\hline SDSSJ121405.11+453818.5 & 2015 Feb 18 & GEMINI-N & $3520-6360$ & 8 & 130.0 & 1.2 & Feige34 & 1.0 & $6 \times 899$ \\
\hline SDSSJ121405.11+453818.5 & 2015 May 18 & MMTO & $3400-8400$ & 8 & 1000.0 & 1.0 & $\mathrm{BD}+284211$ & 1.0 & $3 \times 900$ \\
\hline SDSSJ130234.43+101238.9 & $2013 \mathrm{Feb} 15^{\mathrm{e}}$ & GEMINI-S & $3500-6360$ & 10 & 138.0 & 1.4 & Feige110 & 1.5 & $8 \times 1200$ \\
\hline SDSSJ131445.050-031415.588 & 2013 Mar 09 & GEMINI-S & $3500-6360$ & 10 & 340.0 & 1.1 & Feige110 & 1.5 & $6 \times 1500$ \\
\hline SDSSJ131445.050-031415.588 & 2015 Jan 24 & MMTO & $3400-8400$ & 8 & -22.4 & 1.3 & Feige34 & 1.0 & $4 \times 900$ \\
\hline SDSSJ131445.050-031415.588 & 2015 May 18 & МMTO & $3400-8400$ & 8 & 6.4 & 1.2 & $\mathrm{BD}+284211$ & 1.0 & $2 \times 900$ \\
\hline SDSSJ151421.27+004752.8 & 2013 Mar $10^{\mathrm{e}}$ & GEMINI-S & $3500-6360$ & 10 & 0.0 & 1.3 & Feige110 & 1.5 & $8 \times 1200$ \\
\hline SDSSJ155745.40+554609.7 & 2015 Jan 24 & MMTO & $3400-8400$ & 8 & -113.9 & 1.2 & Feige34 & 1.0 & $2 \times 900$ \\
\hline SDSSJ155745.40+554609.7 & 2015 May 18 & MMTO & $3400-8400$ & 8 & -129.8 & 1.1 & $\mathrm{BD}+284211$ & 1.0 & $4 \times 900$ \\
\hline SDSSJ163800.360+004717.822 & 2013 Apr 08 & GEMINI-S & $3500-6360$ & 10 & 0.0 & 1.3 & Feige110 & 1.5 & $6 \times 1500$ \\
\hline SDSSJ163800.360+004717.822 & 2015 May 18 & MMTO & $3400-8400$ & 8 & 22.0 & 1.2 & $\mathrm{BD}+284211$ & 1.0 & $4 \times 900$ \\
\hline SDSSJ172135.97+294016.0 & 2013 Jun 04 & GEMINI-S & $3500-6360$ & 10 & 180.0 & 2.1 & Feige110 & 1.5 & $6 \times 1500$ \\
\hline SDSSJ172135.97+294016.0 & 2015 May 18 & ММТО & $3400-8400$ & 8 & -77.6 & 1.1 & $\mathrm{BD}+284211$ & 1.0 & $4 \times 900$ \\
\hline SDSSJ181424.13+785402.9 & 2015 Apr 27 & GEMINI-N & $3520-6360$ & 8 & 0.0 & 2.0 & Feige34 & 1.0 & $6 \times 699$ \\
\hline SDSSJ181424.13+785402.9 & 2015 May 18 & МMTO & $3400-8400$ & 8 & -152.3 & 1.5 & $\mathrm{BD}+284211$ & 1.0 & $3 \times 900$ \\
\hline SDSSJ20372.169-051302.964 & $2014 \mathrm{Jul} 14^{\mathrm{e}}$ & GEMINI-S & $3400-6500$ & 10 & 0.0 & 1.1 & Feige110 & 1.5 & $8 \times 1500$ \\
\hline SDSSJ20372.169-051302.964 & 2015 Oct 12 & MMTO & $3400-8400$ & 8 & -8.4 & 1.3 & $\mathrm{BD}+284211$ & 1.25 & $3 \times 1200$ \\
\hline SDSSJ210150.65-054550.9 & 2014 Jul 20 & GEMINI-S & $3400-6500$ & 10 & 0.0 & 1.2 & Feige110 & 1.5 & $6 \times 1300$ \\
\hline SDSSJ210150.65-054550.9 & 2015 May 18 & МMTO & $3400-8400$ & 8 & -39.9 & 1.5 & $\mathrm{BD}+284211$ & 1.0 & $2 \times 900$ \\
\hline SDSSJ210150.65-054550.9 & 2015 Oct 11 & МMTO & $3400-8400$ & 8 & 1.7 & 1.3 & $\mathrm{BD}+284211$ & 1.25 & $6 \times 1200$ \\
\hline SDSSJ232941.330+001107.755 & 2015 Oct $01^{\mathrm{e}}$ & GEMINI-N & $3520-6360$ & 8 & 12.0 & 1.2 & $\mathrm{BD}+284211$ & 1.0 & $11 \times 1099$ \\
\hline SDSSJ232941.330+001107.755 & 2015 Oct 11 & MMTO & $3400-8400$ & 8 & -14.2 & 1.2 & $\mathrm{BD}+284211$ & 1.25 & $6 \times 1200$ \\
\hline SDSSJ235144.29+355542.6 & 2015 May 18 & MMTO & $3400-8400$ & 8 & -74.2 & 1.7 & $\mathrm{BD}+284211$ & 1.0 & $900+765$ \\
\hline SDSSJ235144.29+355542.6 & 2015 Sep 15 & GEMINI-N & $3520-6360$ & 8 & 180.0 & 1.2 & $\mathrm{BD}+284211$ & 1.0 & $6 \times 999$ \\
\hline SDSSJ235144.29+355542.6 & 2015 Oct 11 & MMTO & $3400-8400$ & 8 & 126.4 & 1.0 & $\mathrm{BD}+284211$ & 1.25 & $5 \times 1200$ \\
\hline
\end{tabular}

Notes.

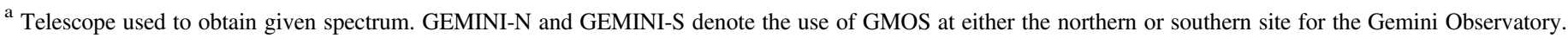
MMTO denotes the use of the Blue Channel spectrograph at the MMT Observatory.

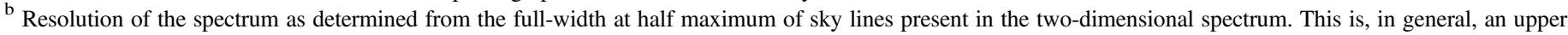

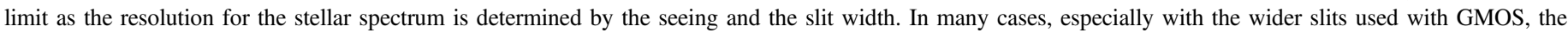
resolution of the spectrum is better than this reported value.

${ }^{\mathrm{c}}$ The position angle of the observations. Spectra from the MMT were typically observed at the parallactic angle, while the Gemini data were not.

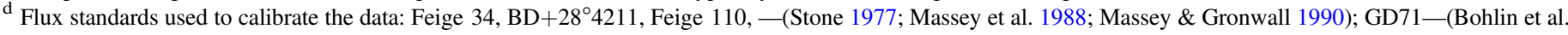
1995); G191B2B-(Oke 1974; Massey et al. 1988).

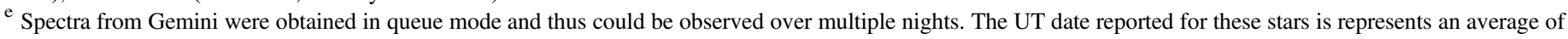
the actual dates. Note that observations for SDSSJ081508 were separated by 10 months.

CCD data and optimally extract (Horne 1986) the spectra. The wavelength scale was evaluated via polynomial fits to calibration lamp spectra and we then resampled the WD spectra onto a linear scale with $1 \AA$ pixel and $2 \AA$ pixel for the GMOS and MMT data, respectively. We used our own custom IDL routines to flux calibrate the data (Matheson et al. 2008). 


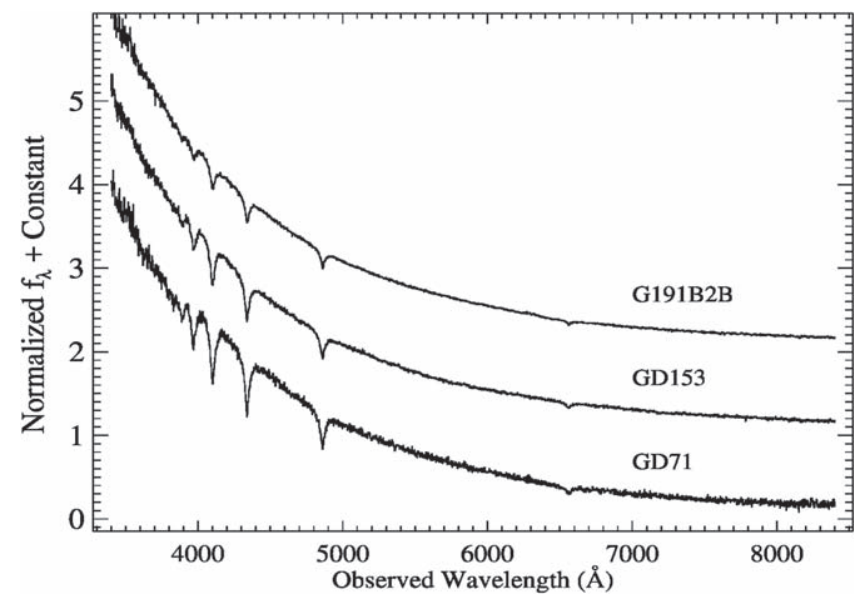

Figure 12. Spectra of the three HST primary CALSPEC standards.

The standard stars for each spectrum are listed in Table 5. The spectra of our DAWDs are shown in three figures. Figure 12 shows the spectra of the three primary CALSPEC standards. The spectra of stars obtained at Gemini are shown in Figure 13 while those obtained at the MMT are shown in Figure 14. The details of the determination of $T_{\text {eff }}$ and $\log g$ will be described in a forthcoming analysis paper (Narayan et al. 2018, in preparation).

One of the WD stars showed indications of abnormality in its spectra. SDSSJ20372.169-051302.964 was observed over several nights with GMOS-S. There is a narrow emission feature present in the cores of the Balmer absorption lines. The emission feature moves relative to the broader line. This may be the result of a low-luminosity companion or some other activity associated with the WD. Thus, this star is unsuitable for use as a spectrophotometric standard because the model spectra only apply to single, inactive DAWDs.

\section{Setting the Photometric Reference System}

The photometry for our candidate spectrophotometric standards needs to be placed on a common flux scale at the top of the atmosphere. To achieve this goal, we observed the three HST primary CALSPEC WDs and the candidate DAWDs by using the same instrument and telescope set-up in Cycle 22 . These observations allowed us to determine the instrumental ZPs for each WFC3 filter and to tie the magnitudes of all of the targets to the same photometric system.

As a first step, we calculated fluxes and magnitudes in the AB photometric system for the HST primary CALSPEC WDs by using the HST tool Pysynphot. ${ }^{16}$ For these simulations, we used the latest model spectra of the three DAWDs provided by the CALSPEC database (mod_010), which are calculated with the Non-Local Thermal-Equilibrium code from Rauch et al. (2013). These models are normalized to an absolute flux level, as defined by the flux of $3.44 \times 10^{-9} \mathrm{erg} \mathrm{cm}^{-2} \mathrm{~s}^{-1} \AA^{-1}$ for Vega at $0.5556 \mu \mathrm{m}$, as reconciled with the $M S X$ mid-IR absolute flux measures (B14).

The AB magnitude system (Oke 1974) is defined for monochromatic fluxes. If the flux at frequency $\nu$ is denoted by $f_{\nu}$ and expressed in units of erg $\mathrm{cm}^{-2} \mathrm{~s}^{-1} \mathrm{~Hz}^{-1}$, then the

\footnotetext{
16 http://pysynphot.readthedocs.io/en/latest/using_pysynphot.html
}

corresponding $\mathrm{AB}$ magnitude at $\nu$ is defined by:

$$
m\left(\mathrm{AB}_{\nu}\right)=-2.5 \log \left(f_{\nu}\right)-48.60 .
$$

This corresponds to a normalization where an object with a flat spectrum has $\mathrm{AB}$ magnitude equal to its $V$ band magnitude (Oke \& Gunn 1983).

To incorporate the idea of $\mathrm{AB}$ magnitudes for nonmonochromatic use, say for a passband $X$, we use the extension as proposed by Fukugita et al. (1996) for a photon proportional detector system to define the quantity $f_{X}$ :

$$
f_{X}=\frac{\int f_{\nu} \nu^{-1} R d \nu}{\int \nu^{-1} R d \nu}=\frac{\int N_{\nu} R d \nu}{\int(h \nu)^{-1} R d \nu}
$$

where $R$ is the (telescope + instrument + filter) response function for passband $X, N_{\nu}$ is the count rate of photons per unit frequency and $h$ is Planck's constant. The numerator on the rightmost side is the photon count rate in the band, so $f_{X}$ is directly proportional to the photon count rate.

The AB magnitude for passband $X$ is then given by:

$$
m\left(\mathrm{AB}_{X}\right)=-2.5 \log \left(f_{X}\right)-48.60 .
$$

A characteristic wavelength, pivot wavelength, is defined to transform flux densities from the frequency to the wavelength domain as:

$$
\lambda_{p}=\sqrt{\frac{c f_{\nu}}{f_{\lambda}}}=\sqrt{\frac{\int R \lambda d \lambda}{\int R \frac{d \lambda}{\lambda}}}
$$

which is a source independent quantity. The Space Telescope (ST) magnitude system is defined in the wavelength domain for passband $X$ as:

$$
m\left(\mathrm{ST}_{X}\right)=-2.5 \log \left(f_{X}\right)-21.10
$$

where ST mag $=0$ is $3.63 \times 10^{-9} \mathrm{erg} \mathrm{cm}^{-2} \mathrm{~s}^{-1} \AA^{-1}$. Having defined $\lambda_{p}$, we can then convert $\mathrm{AB}$ to ST magnitudes with the relation:

$$
m\left(\mathrm{ST}_{\lambda}\right)=m\left(\mathrm{AB}_{\nu}\right)+5 \log \left(\lambda_{p}\right)-18.70 .
$$

We used Pysynphot to calculate synthetic fluxes and magnitudes in the $\mathrm{AB}$ photometric system for the primary WDs. As a reference, we used the most updated files available on the Space Telescope database. ${ }^{17}$ These reference files give the transmission curves for every element in the optical path of the $(H S T+\mathrm{WFC} 3+$ filter $)$ system. For a complete list of the reference files, please see the linked web-page. The AB fluxes and magnitudes obtained for the three HST primary CALSPEC WDs are listed in Table 6.

The derived $\mathrm{AB}$ synthetic magnitudes are compared to the instrumental magnitudes measured from our observations in Cycle 22 for the primary WDs. Figure 15 shows the difference between synthetic and instrumental magnitudes as a function of the observing epoch for the three stars. Observations were divided in multiple exposures for a total of three visits per star in a time interval of $\sim 1.3 \mathrm{yr}$. We performed a $1.5 \sigma$ clipping on the data and we estimated the biweight mean of the difference for the three primary WDs. This difference sets the ZP for our observations. The estimated ZPs with their errors are labeled in each panel of Figure 15. For observations in the F275W and F336W filters, GD71 measurements (black dots) are

\footnotetext{
17 http://www.stsci.edu/hst/observatory/crds/throughput.html
} 


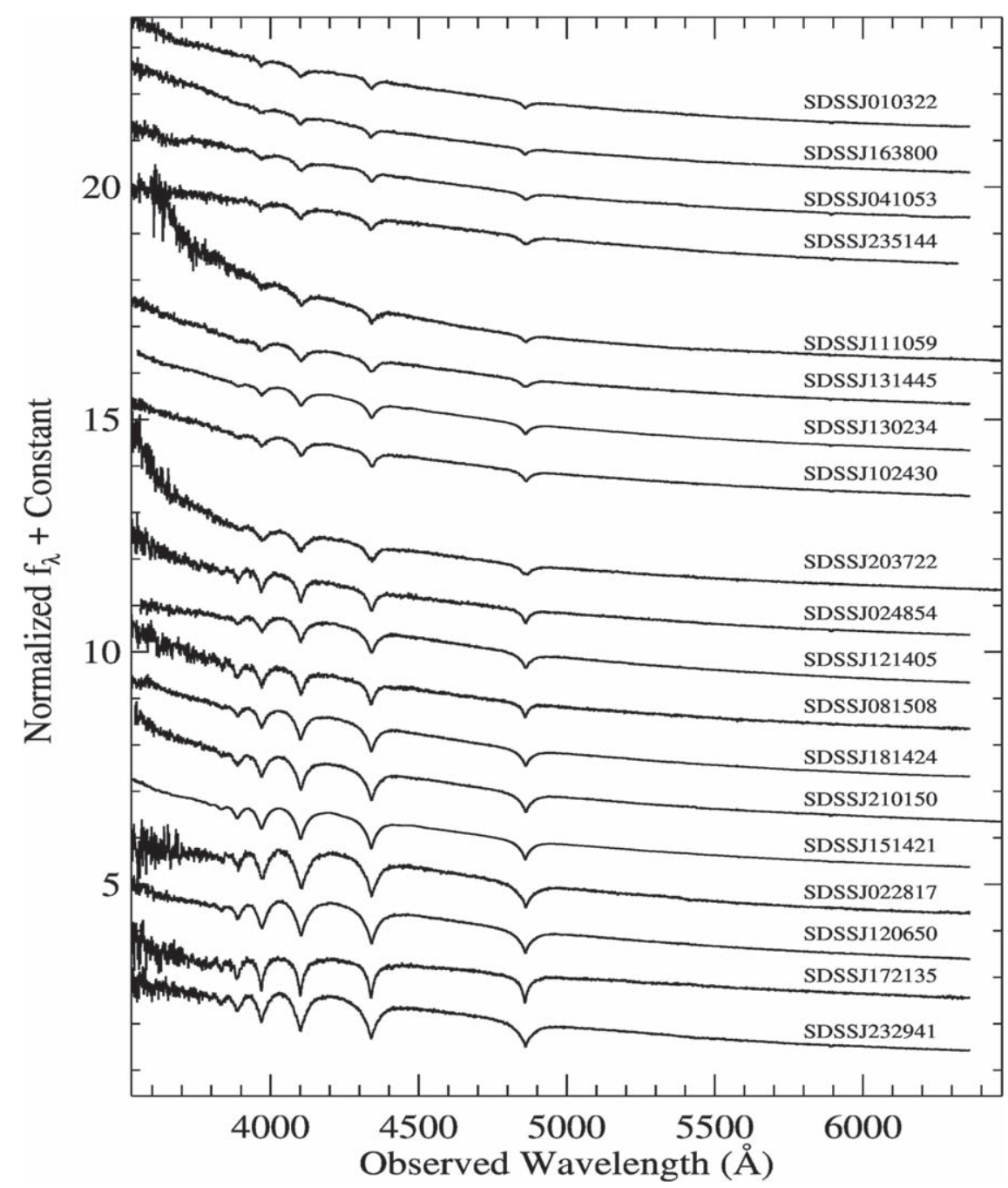

Figure 13. Spectra of the DAWD stars in our program obtained with the GMOS instruments at Gemini. Note that the unusual shapes of some of the spectra are caused by atmospheric dispersion effects and slit losses. Spectra are ordered by $T_{\text {eff }}$, with the hottest stars at the top.

consistently offset; i.e., fainter, compared to the other two WDs (cyan stars and magenta triangles). The cause of this difference is not clear. A set of ZPs for each of the photometric reduction method was estimated and they are listed in Table 7.

As a sanity check we also derived ZPs for the same filters but for an aperture radius of 10 pixels for WFC3-UVIS, and to infinity for WFC3-UVIS and WFC3-IR; i.e., the aperture radii used by the WFC3 team to provide the official ZPs. To derive ZPs to infinity we used the encircled energy correction tables provided by the WFC3 database. ${ }^{18}$

Figure 16 shows the comparison between WFC3 official ZPs and the ZPs measured using our observations, reduced with DAOPHOT, for the three CALSPEC standards as a function of wavelength. Error bars show uncertainties in our ZP estimates because there are no errors provided for the WFC3 ZPs. The left-hand panel shows the comparison for ZPs derived for an aperture radius of 10 pixels (F160W is excluded because ZPs for WFC3-IR are not provided for this aperture), while the right panel shows the same comparison for all filters and for an infinite aperture radius. The two sets of ZPs agree very well, with only the F275W and the F160W filters being $\gtrsim 1 \%$ off.

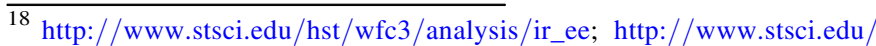
hst/wfc3/analysis/uvis_ee
}

WFC3 official ZPs are calculated by using a set of observations taken between 2009 and 2015: the epoch to which these sensitivities are normalized is then $\approx 2012.5$, and they are an average of measurements collected on the UVIS2 amplifiers C and D. The WFC3 inverse sensitivities change with time. Our ZPs are provided for the average epoch of the observations, i.e., $\approx 2015.5$, and they are based on data collected only on amplifier C. The change in sensitivity of the (detector+filter) system will be analyzed in Section 7.1. In spite of these issues, the overall average difference between the two sets of $\mathrm{ZP}$ is $0.003 \mathrm{mag}$ with a dispersion of $0.005 \mathrm{mag}$ for a 10 pixel aperture, and $0.002 \mathrm{mag}$ with a dispersion of $0.006 \mathrm{mag}$ for the infinite aperture. The ZPs for a 10 pixel aperture radius and for infinity are listed in Table 8 . These ZPs can be used by any astronomer performing observations by using WFC3-UVIS2 and WFC3-IR. They can also be used to tie their photometry to the HST photometric scale.

\subsection{Tracking WFC3 Sensitivity Variation with Time}

We used observations of the three HST primary CALSPEC standards to track the variation of WFC3 sensitivity as a function of filter and time. The observations of the CALSPEC stars span a time interval of approximately $1.3 \mathrm{yr}$, from the fall 


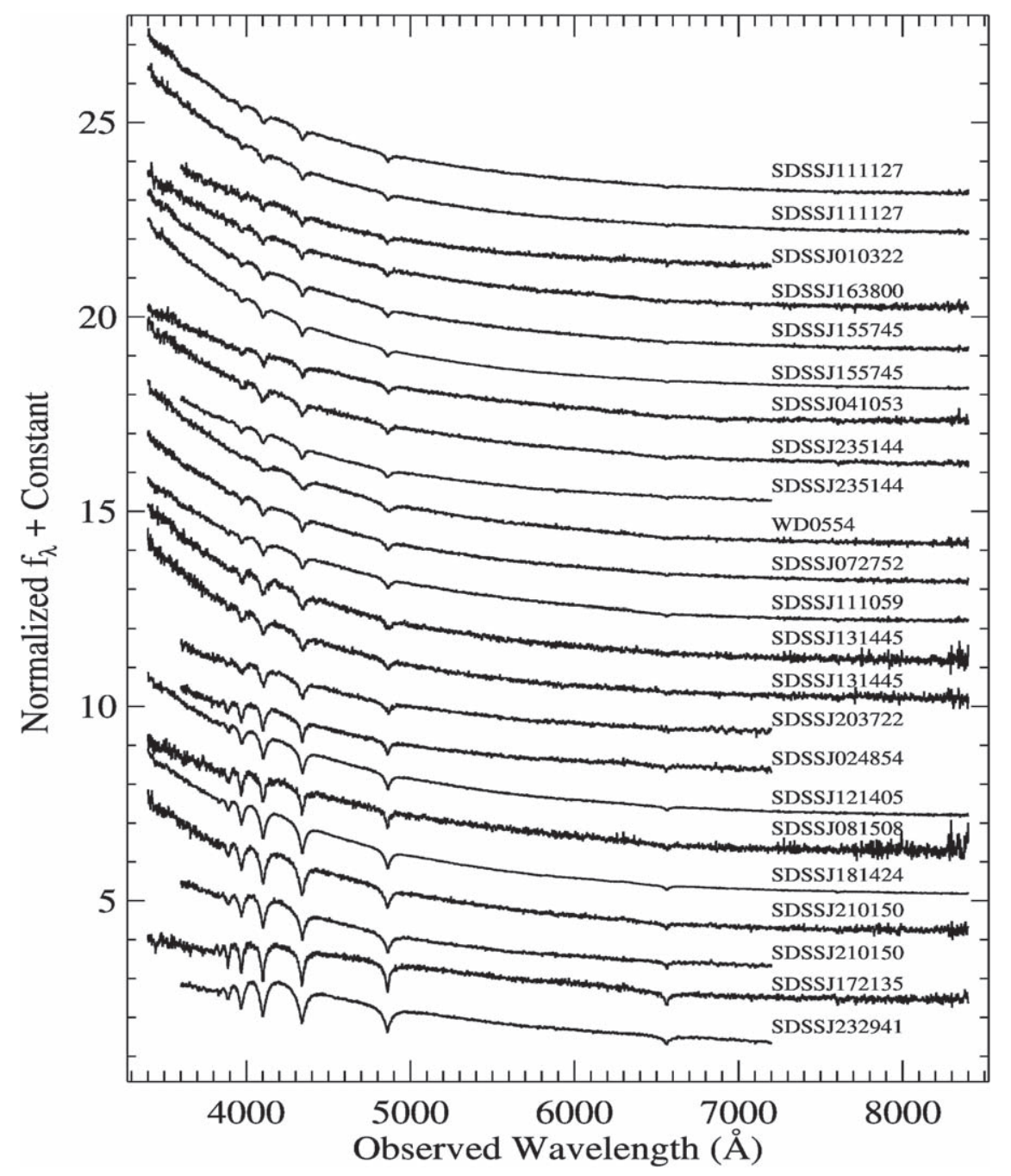

Figure 14. Spectra of the WD stars in our program obtained with the Blue Channel spectrograph at the MMT. Spectra are ordered by $T_{\text {eff }}$, with the hottest stars at the top.

of 2014 to the beginning of 2016. Instrumental count rates for aperture radii 7.5 (WFC3-UVIS) and 5 (WFC3-IR) pixels in the $\mathrm{AB}$ photometric system were derived for the CALSPEC WDs in the six filters for the three different visits, each one with a number of observations ranging from six to eight, depending on the filter; as described in Section 3. Synthetic count rates were derived with Pysynphot for the same stars as observed with WFC3. We followed the same procedure described in Section 7 and we simulated count rates for aperture radii of 7.5 (WFC3-UVIS) and 5 (WFC3-IR) pixels; i.e., the radii that we used to perform photometry on the real images.

Figure 17 shows the ratio of the observed to synthetic count rates as a function of the observing epoch for the three primary WDs $\quad$ (GD71 = black dots, GD153 = cyan stars, and G191B2B = magenta triangles) and the six filters, after we performed a $1.5 \sigma$ clipping of the data. The plot shows that the sensitivity decreases with time for all filters: the decrease is steeper for the bluer filters, F275W, F336W, and F475W; and it is shallower for the redder filters, F625W, F775W, and F160W. We performed a linear least-square fit and obtained slopes ranging from -0.03 to -0.27 , with the larger slopes for
F336W and F475W and the smaller for F625W and F160W. The fit to the data and the final sensitivity decrease rate per year are shown in Figure 17.

The sensitivity loss rates that we obtained from our observations are in good agreement, within uncertainties, with the rates provided by the WFC 3 photometric contamination monitor studies. One of the contamination monitor programs is based on about $8 \mathrm{yr}$ of observations of the CALSPEC WD GWR70. These data show that WFC3 sensitivity decreases by less than $0.01 \%$ for the UV filters F275W and F336W (see Table 4 of Shanahan et al. 2017 for more details). However, no measurements are available for the F475W, F625W and F775W filters from this contamination monitor. It is worth mentioning that UV filters had an increase in sensitivity soon after WFC3 was installed and they then started to decrease (see Figure 8 in Shanahan et al. 2017). The very low percentage decrease obtained by Shanahan et al. (2017) for the UV filters is due to fitting all of the measurements for GWR70 at the same time. A more recent contamination monitor study from the WFC3 team that was based on $8 \mathrm{yr}$ of photometry for the three primary WDs and the CALSPEC G-type standard P330E was able to obtain steeper slopes for the UV filters by only 
Table 6

Synthetic Magnitudes and Fluxes in the AB and ST Photometric System for the Three HST Primary CALSPEC DAWDs as Simulated by Using Pysynphot

\begin{tabular}{|c|c|c|c|c|c|}
\hline Filter & $\begin{array}{l}\lambda_{p} \\
(\AA)\end{array}$ & $\begin{array}{c}\text { AB mag } \\
\text { (mag) }\end{array}$ & $\begin{array}{c}F_{\nu} \\
\left(\operatorname{erg~cm}{ }^{-2}\right. \\
\left.\mathrm{s}^{-1} \mathrm{~Hz}^{-1}\right)\end{array}$ & $\begin{array}{l}\text { ST mag } \\
\text { (mag) }\end{array}$ & $\begin{array}{c}F_{\lambda} \\
\left(\text { erg } \mathrm{cm}^{-2}\right. \\
\left.\mathrm{s}^{-1} \mathrm{~Hz}^{-1}\right)\end{array}$ \\
\hline \multicolumn{6}{|c|}{ GD153 } \\
\hline F275W & 2703 & 12.200 & $4.78 \mathrm{e}-25$ & 10.669 & $1.96 \mathrm{e}-13$ \\
\hline F336W & 3354 & 12.566 & $3.41 \mathrm{e}-25$ & 11.503 & $9.09 \mathrm{e}-14$ \\
\hline F475W & 4770 & 13.098 & $2.09 \mathrm{e}-25$ & 12.799 & $2.76 \mathrm{e}-14$ \\
\hline F625W & 6240 & 13.598 & $1.32 \mathrm{e}-25$ & 13.882 & $1.02 \mathrm{e}-14$ \\
\hline F775W & 7651 & 14.004 & $9.09 \mathrm{e}-26$ & 14.730 & $4.66 \mathrm{e}-15$ \\
\hline F160W & 15,369 & 15.414 & $2.48 \mathrm{e}-26$ & 17.654 & $3.15 e-16$ \\
\hline \multicolumn{6}{|c|}{ GD71 } \\
\hline F275W & 2703 & 11.981 & $5.85 e-25$ & 10.450 & $2.40 \mathrm{e}-13$ \\
\hline F336W & 3354 & 12.327 & $4.26 \mathrm{e}-25$ & 11.264 & $1.13 \mathrm{e}-13$ \\
\hline F475W & 4770 & 12.794 & $2.77 \mathrm{e}-25$ & 12.496 & $3.64 \mathrm{e}-14$ \\
\hline F625W & 6240 & 13.275 & $1.78 \mathrm{e}-25$ & 13.558 & $1.37 \mathrm{e}-14$ \\
\hline F775W & 7651 & 13.672 & $1.23 e-25$ & 14.398 & $6.32 \mathrm{e}-15$ \\
\hline F160W & 15,369 & 15.060 & $3.43 e-26$ & 17.301 & $4.36 e-16$ \\
\hline \multicolumn{6}{|c|}{ G191B2B } \\
\hline F275W & 2703 & 10.492 & $2.30 \mathrm{e}-24$ & 8.960 & $9.46 \mathrm{e}-13$ \\
\hline F336W & 3354 & 10.892 & $1.60 \mathrm{e}-24$ & 9.829 & $4.25 \mathrm{e}-13$ \\
\hline F475W & 4770 & 11.500 & $9.12 \mathrm{e}-25$ & 11.201 & $1.20 \mathrm{e}-13$ \\
\hline F625W & 6240 & 12.030 & $5.60 \mathrm{e}-25$ & 12.314 & $4.31 \mathrm{e}-14$ \\
\hline F775W & 7651 & 12.449 & $3.81 \mathrm{e}-25$ & 13.175 & $1.95 \mathrm{e}-14$ \\
\hline F160W & 15,369 & 13.885 & $1.01 \mathrm{e}-25$ & 16.125 & $1.29 \mathrm{e}-15$ \\
\hline
\end{tabular}

Note. The pivot wavelength for each filter is also listed. See text for more details.

considering measurements from when the sensitivity started to decrease. The new sensitivity loss rates range from $\sim-0.05$ to $-0.2 \%$ per year for the UVIS filters F275W, F336W, F475W, F625W, and F775W (Khandrika et al. 2018). These results will soon published be in a WFC3 ISR.

The WFC3 sensitivity loss rates that we derived by using our observations of the primary WDs have very large errors, $0.1 \%-$ $0.2 \%$. Our data cover a very short time interval of a little more than $1 \mathrm{yr}$ and they are insufficient to fully characterize the sensitivity variations with time. Meanwhile, the total dispersion of the measurements for the three primary WDs is always less than $\sim 0.005$ mag in all UVIS filters and less then $1 \%$ for in the infrared in the considered time interval of our program observations. Therefore, we did not apply any time correction to the photometry.

\subsection{The Final Magnitudes}

The ZPs obtained in Section 7 were applied to the weighted mean instrumental magnitudes of all the 23 candidate standard DAWDs. The ZPs were derived by using observations of the three HST primary CALSPEC standards, performed under the same conditions, and reduced with the same technique. Therefore, they take into account any possible systematics in the observations and data reduction process. For each of the three different reduction methods, final calibrated magnitudes for filter $X$ in the $\mathrm{AB}$ photometric system are derived as:

$$
\begin{aligned}
m\left(\mathrm{AB}_{X}\right)_{\text {cal }} & =m\left(\mathrm{AB}_{X}\right)_{\text {inst }}+\mathrm{ZP}_{\mathrm{AB}_{X}} \\
& =\left(-2.5 \log \left(f_{X}\right)-48.60\right)+\mathrm{ZP}_{\mathrm{AB}_{X}}
\end{aligned}
$$

where $f_{X}$ is in electrons/s, for aperture radii of 7.5 (WFC3UVIS) and 5 pixels (WFC3-IR), respectively, and $\mathrm{ZP}_{\mathrm{AB}_{X}}$ are the estimated ZPs listed in Table 7.

The final magnitudes for the 23 candidate spectrophotometric standard DAWDs and the HST primary CALSPEC WDs are listed in Table 9. Figure 3 shows the $B p-R p$, F475W - F775W color-color diagram for the 23 candidate spectrophotometric standard DAWDs, where GAIA and WFC3 magnitudes derived with DAOPHOT are plotted.

Regardless of the photometric reduction method used, the magnitudes for our DAWDs have an average dispersion ranging from 1 to 3 milli-mag for the WFC3-UVIS filters and from 5 to 10 milli-mag for the F160W IR filter.

\section{Summary and Conclusions}

In this paper, we presented the methods used to provide subpercent precision photometry for a set of faint candidate spectrophotometric standard DAWDs. We also presented data reduction processes and possible sources of uncertainties of the spectroscopic data collected for the same stars. These spectra are used to derive temperature and surface gravities for the candidate standards.

To investigate the possible sources of systematics and to derive reliable uncertainties for the DAWD photometry, we used three different software packages to reduce WFC3 data: DAOPHOT, ILAPH, and SExtractor. Our analysis showed that photometry performed with the first two packages agrees very well within uncertainties. Meanwhile, photometry from SExtractor shows larger dispersion and a trend where the magnitudes of fainter stars are fainter when compared to DAOPHOT and ILAPH magnitudes, particularly in the bluest filters: F275W and F336W. This trend is probably due to SExtractor over-estimating the sky background.

We tested our data for photometric uncertainties due to the presence of external or internal persistence on the IR images. We found that the largest fraction of pixels affected by a persistence signal higher than $0.01 \mathrm{e}^{-} \mathrm{s}^{-1}$ is $0.33 \%$, for images of star SDSSJ181424.075+785403.048. However, the affected pixels do not overlap with the location of the star on the images. Our observing strategy was devised to avoid selfpersistence in our exposures.

CRNL in WFC3-IR exposures was estimated to be $0.010 \pm 0.0025 \mathrm{mag}$ per dex, which might slightly affect our observations. However, we do not apply any CRNL corrections on the photometry presented here and we plan to fully characterize this effect in NA19.

Our data show no systematics in the photometry due to the WFC3-UVIS shutter shading effect for an aperture radius of 7.5 pixels. We tested photometry on $1 \mathrm{~s}$ exposures for G191B2B and we found that the dispersion of the measurements on images collected by using shutter blade B, $\sigma=0.004 \mathrm{mag}$, is about the same when compared to the dispersion on images observed with blade A, $\sigma=0.005$ mag. All the other DAWDs were observed with exposure times $\gtrsim 5 \mathrm{~s}$. Consequently, our observations are not affected by shutter shading.

The presence of unseen companion stars could also introduce uncertainties/systematics in the photometry. However, the observed DAWDs are all in very sparse fields, with a maximum of three other objects, including the DAWD in the $\sim 20 \times 20^{\prime \prime}$ observed FoV down to $g \sim 23 \mathrm{mag}$. Our simulations also showed that stars fainter than 6 mag compared to the 

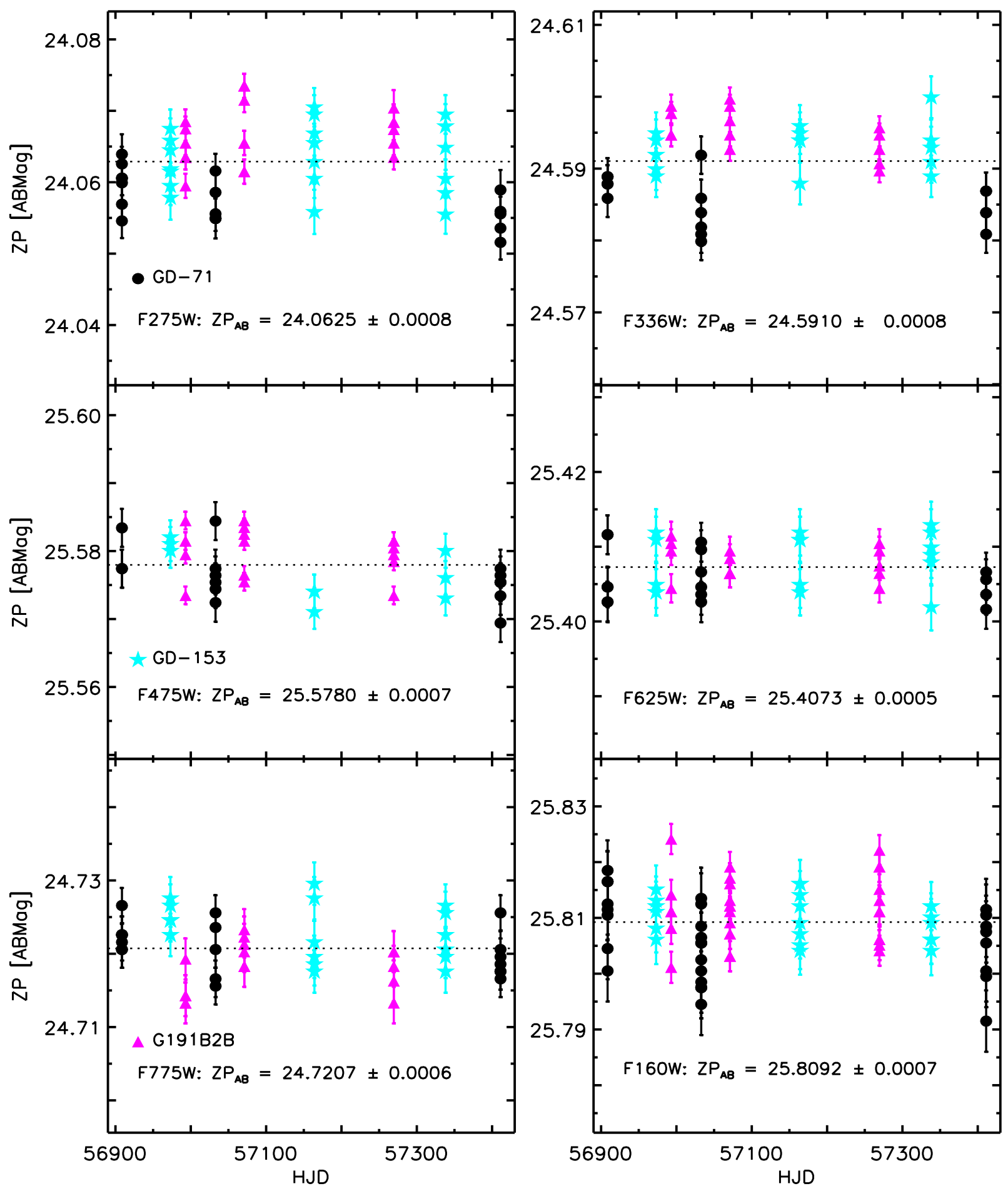

Figure 15. ZPs in the AB photometric system based on all the observations for the three $H S T$ primary CALSPEC WDs (GD71 $=$ black dots, GD153 $=$ cyan stars, and G191B2B = magenta triangles) as a function of the Heliocentric Julian date (HJD) for six WFC3-UVIS and WFC3-IR filters as measured with DAOPHOT. Error bars are shown and the derived ZPs are labeled.

target WDs cannot affect the photometry of the target DAWD, even if they fall inside the aperture radius. Therefore, we can safely assume that the photometry of our set of standards cannot be contaminated by unseen neighbor stars.

Time-series observations collected with the LCO network of telescopes showed that most of our candidate spectrophotometric standards are stable. Two of them-namely
SDSSJ20372.169-051302.964 and WD0554-165-show clear signs of variability in their light curves. The first star also shows emission features in the Balmer lines of the spectra, which implies the presence of a low-mass companion. We do not know the origin of the variability for WD0554-165. Two other DAWDs-SDSSJ010322.10-002047.7 and SDSSJ102430.93-003207.0— show hints of variability, but 


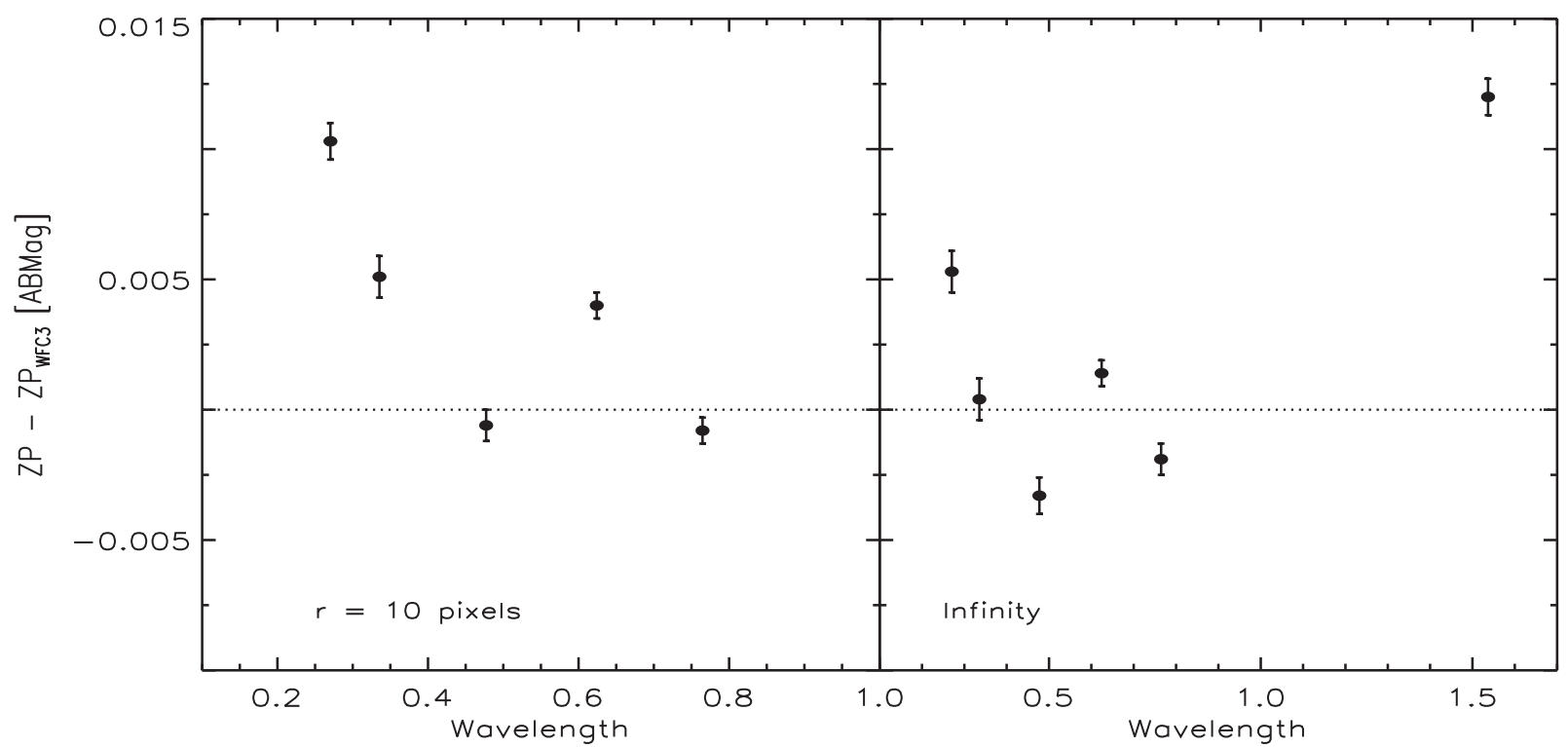

Figure 16. Comparison between ZPs in the AB photometric system measured from our observations of the three HST primary CALSPEC WDs and the WFC 3 official ZPs as a function of wavelength. The ZPs are derived for an aperture radius of 10 pixels (left panel, UVIS) and infinity (right, UVIS + IR). See text for more details.

Table 7

Zero Points and their Uncertainties for WFC3-UVIS (Aperture Radius $r=7.5$ pixels) and IR ( $r=5$ pixels) Observations in the AB Photometric System

\begin{tabular}{lccccc}
\hline \hline Filter & $\begin{array}{c}\text { ZP (DAOPHOT) } \\
(\mathrm{mag})\end{array}$ & $\begin{array}{c}\text { eZP (DAOPHOT) } \\
(\mathrm{mag})\end{array}$ & $\begin{array}{c}\text { ZP (ILAPH) } \\
(\mathrm{mag})\end{array}$ & $\begin{array}{c}\text { eZP (ILAPH) } \\
(\mathrm{mag})\end{array}$ & $\begin{array}{c}\text { ZP (Sextractor) } \\
(\mathrm{mag})\end{array}$ \\
\hline F275W & 24.0612 & 0.0008 & 24.0596 & 0.0009 & 24.0594 \\
F336W & 24.5910 & 0.0008 & 24.5899 & 0.0008 & 0.0009 \\
F475W & 25.5780 & 0.0007 & 25.5774 & 0.0009 & 24.5889 \\
F625W & 25.4073 & 0.0005 & 25.4056 & 0.0007 & 25.5761 \\
F775W & 24.7207 & 0.0006 & 24.7189 & 0.0008 & 25.4043 \\
F160W & 25.8092 & 0.0007 & 25.8116 & 0.0009 & 24.7171 \\
& & & & 25.8106 & 0.0007 \\
\end{tabular}

Note. Zero points are derived by using observations of the three HST primary calspec standards as measured with three different methods.

Table 8

Zero Points and their Uncertainties for 10 pixel Aperture Radius (WFC3-UVIS) and Infinity (WFC3-UVIS, WFC3-IR) in the AB Photometric System

\begin{tabular}{|c|c|c|c|c|c|c|}
\hline Filter & $\begin{array}{l}\mathrm{ZP}_{10} \\
(\mathrm{mag})\end{array}$ & $\begin{array}{l}\mathrm{eZP}_{10} \\
(\mathrm{mag})\end{array}$ & $\begin{array}{l}\mathrm{ZP}_{\text {inf }} \\
(\mathrm{mag})\end{array}$ & $\begin{array}{l}\mathrm{eZP}_{\text {inf }} \\
(\mathrm{mag})\end{array}$ & $\begin{array}{c}\mathrm{ZP}_{10}(\mathrm{WFC} 3) \\
(\mathrm{mag})\end{array}$ & $\begin{array}{c}\mathrm{ZP}_{\text {inf }}(\mathrm{WFC} 3) \\
(\mathrm{mag})\end{array}$ \\
\hline F275W & 24.0853 & 0.0007 & 24.2293 & 0.0008 & 24.075 & 24.224 \\
\hline F336W & 24.6131 & 0.0008 & 24.7344 & 0.0008 & 24.608 & 24.734 \\
\hline F625W & 25.4310 & 0.0005 & 25.5334 & 0.0005 & 25.427 & 25.532 \\
\hline F775W & 24.7522 & 0.0005 & 24.8571 & 0.0006 & 24.753 & 24.859 \\
\hline F160W & $\ldots$ & $\ldots$ & 25.9580 & 0.0007 & $\ldots$ & 25.946 \\
\hline
\end{tabular}

Note. Zero points are derived by using our observations of the three HST primary CALSPEC standards as measured with DAOPHOT (first columns) and the official WFC3 values are in the last two columns (Current WFC3 UVIS and IR official ZPs can be found at http://www.stsci.edu/hst/wfc3/phot_zp_lbn).

these results need to be confirmed with further data. SDSSJ20372.169-051302.964 and WD0554-165 will be excluded from our set of candidate standard DAWDs.

We used observations of the three HST primary CALSPEC standards, which were collected at the same time as our target DAWDs, to estimate ZPs in the AB photometric system to be applied to instrumental magnitudes of all the observed targets.

We also derived ZPs in the AB photometric system for six WFC3 filters-namely F275W, F336W, F475W, F625W, F775W and F160W-for a 10 pixel aperture radius and for infinity. The ZPs are provided in Table 8 and they can be used to calibrate any WFC3-UVIS2 photometry.

We also verified for the presence of WFC3 sensitivity changes during the $\sim 1.3 \mathrm{yr}$ of the observations by using the same data. A decrease in sensitivity is observed in all six filters, with the largest percentage decline in sensitivity for $\mathrm{F} 475 \mathrm{~W}$ $(-0.27 \%)$ and the smallest for F625W and F160W (-0.03\%). Although our data do not span a sufficient time interval to fully characterize WFC3 sensitivity behavior, the overall dispersion of the measurements over the time interval of our observations 


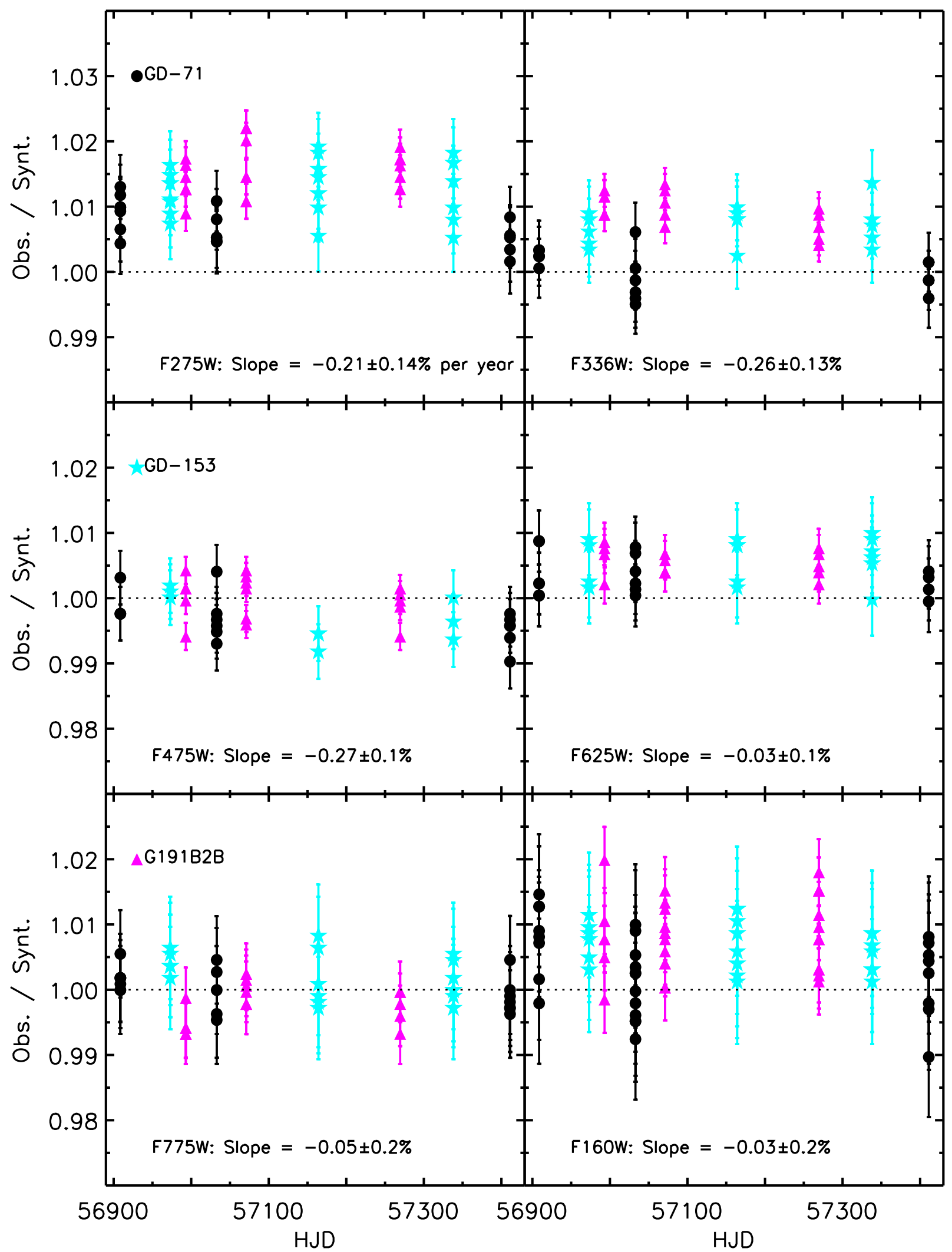

Figure 17. Ratio of observed to synthetic count rates for the three HST primary CALSPEC standards $($ GD71 = black dots, GD153= cyan stars, and G191B2B = magenta triangles) as a function of the Heliocentric Julian date (HJD) for six WFC3-UVIS and WFC3-IR filters. Error bars and the slope fits are shown. The rate of yearly sensitivity loss is labeled.

is less than $0.5 \%$ for WFC3-UVIS and less than $1 \%$ for WFC3IR. Therefore, we do not apply any time correction to our photometry.
We provided final calibrated $\mathrm{AB}$ magnitudes in five WFC3UVIS filters and one IR filter for the 23 candidate spectrophotometric standard DAWDs and the three HST primary 
Table 9

Photometry in the WFC3 UVIS and IR Filters for the 3 HST Primary CALSPEC Standards and the 23 Candidate Standard DAWDs in the AB Photometric System

\begin{tabular}{|c|c|c|c|c|c|c|c|c|c|c|c|c|}
\hline Star & $\begin{array}{c}\text { F275W } \\
(\mathrm{mag})\end{array}$ & $\begin{array}{c}\mathrm{dF} 275 \mathrm{~W} \\
\text { (mag) }\end{array}$ & $\begin{array}{c}\text { F336W } \\
\text { (mag) }\end{array}$ & $\begin{array}{c}\mathrm{dF} 336 \mathrm{~W} \\
\text { (mag) }\end{array}$ & $\begin{array}{c}\text { F475W } \\
(\mathrm{mag})\end{array}$ & $\begin{array}{c}\mathrm{dF} 475 \mathrm{~W} \\
\text { (mag) }\end{array}$ & $\begin{array}{c}\text { F625W } \\
(\mathrm{mag})\end{array}$ & $\begin{array}{c}\mathrm{dF} 625 \mathrm{~W} \\
\text { (mag) }\end{array}$ & $\begin{array}{c}\text { F775W } \\
(\mathrm{mag})\end{array}$ & $\begin{array}{c}\mathrm{dF} 775 \mathrm{~W} \\
\text { (mag) }\end{array}$ & $\begin{array}{c}\text { F160W } \\
(\mathrm{mag})\end{array}$ & $\begin{array}{c}\mathrm{dF} 160 \mathrm{~W} \\
\text { (mag) }\end{array}$ \\
\hline \multicolumn{13}{|c|}{ DAOPHOT } \\
\hline Offsets & $\ldots$ & $\ldots$ & -0.033 & 0.001 & -0.007 & 0.005 & -0.006 & 0.004 & 0.013 & 0.006 & -0.014 & 0.004 \\
\hline G191B2B & 10.488 & 0.002 & 10.888 & 0.001 & 11.498 & 0.001 & 12.030 & 0.001 & 12.451 & 0.001 & 13.883 & 0.002 \\
\hline GD71 & 11.986 & 0.002 & 12.333 & 0.001 & 12.796 & 0.001 & 13.277 & 0.001 & 13.672 & 0.001 & 15.065 & 0.002 \\
\hline GD153 & 12.199 & 0.002 & 12.565 & 0.001 & 13.099 & 0.002 & 13.597 & 0.001 & 14.002 & 0.001 & 15.413 & 0.002 \\
\hline SDSSJ010322.19-002047.7 & 18.191 & 0.004 & 18.524 & 0.006 & 19.082 & 0.005 & 19.562 & 0.005 & 19.967 & 0.005 & 21.364 & 0.020 \\
\hline SDSSJ022817.16-082716.4 & 19.512 & 0.006 & 19.732 & 0.037 & 19.811 & 0.005 & 20.178 & 0.006 & 20.506 & 0.007 & 21.737 & 0.015 \\
\hline SDSSJ024854.96+334548.3 & 17.829 & 0.004 & 18.042 & 0.004 & 18.367 & 0.003 & 18.745 & 0.002 & 19.078 & 0.002 & 20.341 & 0.006 \\
\hline SDSSJ041053.632-063027.580 & 18.110 & 0.009 & 18.401 & 0.004 & 18.879 & 0.004 & 19.254 & 0.003 & 19.387 & 0.007 & 19.500 & 0.005 \\
\hline WD0554-165 & 16.774 & 0.005 & 17.150 & 0.003 & 17.720 & 0.005 & 18.221 & 0.002 & 18.622 & 0.007 & 20.046 & 0.002 \\
\hline SDSSJ072752.76+321416.1 & 17.158 & 0.003 & 17.467 & 0.003 & 17.990 & 0.003 & 18.456 & 0.002 & 18.839 & 0.002 & 20.214 & 0.006 \\
\hline SDSSJ081508.78+073145.7 & 18.939 & 0.005 & 19.262 & 0.006 & 19.713 & 0.004 & 20.186 & 0.004 & 20.578 & 0.005 & 21.967 & 0.015 \\
\hline SDSSJ102430.93-003207.0 & 18.248 & 0.038 & 18.509 & 0.004 & 18.903 & 0.004 & 19.314 & 0.005 & 19.667 & 0.009 & 20.989 & 0.014 \\
\hline SDSSJ111059.42-170954.2 & 17.039 & 0.004 & 17.351 & 0.004 & 17.864 & 0.002 & 18.313 & 0.002 & 18.690 & 0.002 & 20.057 & 0.005 \\
\hline SDSSJ111127.30+395628.0 & 17.432 & 0.004 & 17.832 & 0.005 & 18.419 & 0.003 & 18.940 & 0.004 & 19.344 & 0.002 & 20.795 & 0.010 \\
\hline SDSSJ120650.504+020143.810 & 18.236 & 0.004 & 18.484 & 0.004 & 18.669 & 0.004 & 19.058 & 0.004 & 19.411 & 0.005 & 20.700 & 0.011 \\
\hline SDSSJ121405.11+453818.5 & 16.938 & 0.002 & 17.279 & 0.002 & 17.758 & 0.002 & 18.231 & 0.002 & 18.630 & 0.002 & 20.035 & 0.004 \\
\hline SDSSJ130234.43+101238.9 & 16.185 & 0.002 & 16.519 & 0.002 & 17.033 & 0.002 & 17.512 & 0.002 & 17.904 & 0.001 & 19.302 & 0.004 \\
\hline SDSSJ131445.050-031415.588 & 18.254 & 0.004 & 18.593 & 0.004 & 19.100 & 0.004 & 19.571 & 0.004 & 19.933 & 0.010 & 21.329 & 0.012 \\
\hline SDSSJ151421.27+004752.8 & 15.108 & 0.002 & 15.387 & 0.002 & 15.707 & 0.002 & 16.119 & 0.001 & 16.470 & 0.001 & 17.783 & 0.004 \\
\hline SDSSJ155745.40+554609.7 & 16.496 & 0.002 & 16.873 & 0.002 & 17.468 & 0.003 & 17.990 & 0.002 & 18.389 & 0.002 & 19.832 & 0.005 \\
\hline SDSSJ163800.360+004717.822 & 18.012 & 0.007 & 18.314 & 0.004 & 18.838 & 0.004 & 19.283 & 0.003 & 19.664 & 0.005 & 20.999 & 0.015 \\
\hline SDSSJ172135.97+294016.0 & 20.370 & 0.010 & 20.086 & 0.014 & 19.654 & 0.004 & 19.670 & 0.003 & 19.769 & 0.003 & 20.554 & 0.022 \\
\hline SDSSJ181424.075+785403.048 & 15.788 & 0.002 & 16.119 & 0.002 & 16.542 & 0.002 & 17.004 & 0.002 & 17.392 & 0.001 & 18.782 & 0.002 \\
\hline SDSSJ203722.169-051302.964 & 18.254 & 0.007 & 18.540 & 0.004 & 18.940 & 0.006 & 19.371 & 0.007 & 19.674 & 0.008 & 20.965 & 0.009 \\
\hline SDSSJ210150.65-054550.9 & 18.064 & 0.003 & 18.328 & 0.004 & 18.654 & 0.003 & 19.062 & 0.002 & 19.419 & 0.003 & 20.737 & 0.006 \\
\hline SDSSJ232941.330+001107.755 & 17.940 & 0.003 & 18.105 & 0.004 & 18.158 & 0.005 & 18.472 & 0.003 & 18.785 & 0.006 & 19.997 & 0.007 \\
\hline SDSSJ235144.29+375542.6 & 17.446 & 0.003 & 17.658 & 0.002 & 18.073 & 0.002 & 18.459 & 0.002 & 18.788 & 0.002 & 20.070 & 0.004 \\
\hline \multicolumn{13}{|c|}{ SExtractor } \\
\hline Offsets & $\ldots$ & $\ldots$ & -0.031 & 0.003 & -0.004 & 0.003 & -0.009 & 0.002 & 0.012 & 0.004 & -0.011 & 0.005 \\
\hline G191B2B & 10.488 & 0.002 & 10.889 & 0.001 & 11.497 & 0.001 & 12.029 & 0.001 & 12.451 & 0.001 & 13.884 & 0.001 \\
\hline GD71 & 11.989 & 0.002 & 12.335 & 0.001 & 12.798 & 0.001 & 13.277 & 0.001 & 13.671 & 0.001 & 15.063 & 0.002 \\
\hline GD153 & 12.196 & 0.002 & 12.562 & 0.001 & 13.097 & 0.002 & 13.598 & 0.001 & 14.003 & 0.001 & 15.414 & 0.002 \\
\hline SDSSJ010322.19-002047.7 & 18.197 & 0.004 & 18.532 & 0.005 & 19.088 & 0.005 & 19.564 & 0.004 & 19.968 & 0.005 & 21.359 & 0.010 \\
\hline SDSSJ022817.16-082716.4 & 19.531 & 0.006 & 19.761 & 0.018 & 19.823 & 0.006 & 20.178 & 0.005 & 20.514 & 0.004 & 21.740 & 0.013 \\
\hline SDSSJ024854.96+334548.3 & 17.840 & 0.003 & 18.047 & 0.004 & 18.368 & 0.003 & 18.745 & 0.002 & 19.079 & 0.002 & 20.335 & 0.005 \\
\hline SDSSJ041053.632-063027.580 & 18.109 & 0.009 & 18.410 & 0.004 & 18.884 & 0.004 & 19.256 & 0.003 & 19.387 & 0.005 & 19.500 & 0.005 \\
\hline WD0554-165 & 16.777 & 0.005 & 17.153 & 0.004 & 17.729 & 0.003 & 18.222 & 0.003 & 18.619 & 0.005 & 20.043 & 0.006 \\
\hline SDSSJ072752.76+321416.1 & 17.164 & 0.003 & 17.474 & 0.003 & 17.993 & 0.002 & 18.457 & 0.002 & 18.840 & 0.002 & 20.214 & 0.005 \\
\hline SDSSJ081508.78+073145.7 & 18.965 & 0.007 & 19.280 & 0.005 & 19.714 & 0.005 & 20.185 & 0.004 & 20.579 & 0.005 & 21.967 & 0.012 \\
\hline SDSSJ102430.93-003207.0 & 18.264 & 0.014 & 18.517 & 0.004 & 18.909 & 0.004 & 19.314 & 0.003 & 19.668 & 0.008 & 20.994 & 0.010 \\
\hline SDSSJ111059.42-170954.2 & 17.047 & 0.003 & 17.359 & 0.004 & 17.867 & 0.002 & 18.314 & 0.002 & 18.689 & 0.002 & 20.054 & 0.005 \\
\hline SDSSJ111127.30+395628.0 & 17.437 & 0.004 & 17.838 & 0.005 & 18.424 & 0.003 & 18.940 & 0.004 & 19.346 & 0.002 & 20.790 & 0.008 \\
\hline SDSSJ120650.504+020143.810 & 18.244 & 0.004 & 18.491 & 0.004 & 18.672 & 0.004 & 19.060 & 0.003 & 19.412 & 0.004 & 20.703 & 0.006 \\
\hline
\end{tabular}


Table 9

(Continued)

\begin{tabular}{|c|c|c|c|c|c|c|c|c|c|c|c|c|}
\hline Star & $\begin{array}{c}\text { F275W } \\
(\mathrm{mag})\end{array}$ & $\begin{array}{c}\mathrm{dF} 275 \mathrm{~W} \\
\text { (mag) }\end{array}$ & $\begin{array}{c}\text { F336W } \\
(\mathrm{mag})\end{array}$ & $\begin{array}{c}\text { dF336W } \\
\text { (mag) }\end{array}$ & $\begin{array}{c}\text { F475W } \\
(\mathrm{mag})\end{array}$ & $\begin{array}{c}\mathrm{dF} 475 \mathrm{~W} \\
(\mathrm{mag})\end{array}$ & $\begin{array}{c}\text { F625W } \\
(\mathrm{mag})\end{array}$ & $\begin{array}{c}\text { dF625W } \\
\text { (mag) }\end{array}$ & $\begin{array}{c}\text { F775W } \\
(\mathrm{mag})\end{array}$ & $\begin{array}{c}\mathrm{dF} 775 \mathrm{~W} \\
(\mathrm{mag})\end{array}$ & $\begin{array}{c}\text { F160W } \\
(\mathrm{mag})\end{array}$ & $\begin{array}{c}\mathrm{dF} 160 \mathrm{~W} \\
\text { (mag) }\end{array}$ \\
\hline $\begin{array}{l}\text { SDSSJ121405.11+453818.5 } \\
\text { S. }\end{array}$ & 16.943 & 0.003 & 17.283 & 0.002 & 17.759 & 0.002 & 18.231 & 0.002 & 18.631 & 0.002 & 20.036 & 0.004 \\
\hline SDSSJ130234.43+101238.9 & 16.191 & 0.002 & 16.522 & 0.002 & 17.036 & 0.002 & 17.514 & 0.002 & 17.903 & 0.001 & 19.302 & 0.004 \\
\hline SDSSJ131445.050-031415.588 & 18.258 & 0.004 & 18.600 & 0.005 & 19.106 & 0.004 & 19.572 & 0.004 & 19.936 & 0.008 & 21.327 & 0.008 \\
\hline SDSSJ151421.27+004752.8 & 15.112 & 0.002 & 15.390 & 0.002 & 15.708 & 0.002 & 16.119 & 0.002 & 16.470 & 0.001 & 17.783 & 0.004 \\
\hline SDSSJ155745.40+554609.7 & 16.501 & 0.002 & 16.877 & 0.002 & 17.472 & 0.002 & 17.991 & 0.002 & 18.389 & 0.002 & 19.832 & 0.005 \\
\hline SDSSJ163800.360+004717.822 & 18.013 & 0.007 & 18.321 & 0.004 & 18.842 & 0.004 & 19.285 & 0.003 & 19.663 & 0.004 & 21.002 & 0.007 \\
\hline SDSSJ172135.97+294016.0 & 20.374 & 0.013 & 20.089 & 0.012 & 19.666 & 0.005 & 19.670 & 0.003 & 19.769 & 0.003 & 20.551 & 0.024 \\
\hline SDSSJ181424.075+785403.048 & 15.792 & 0.002 & 16.122 & 0.002 & 16.543 & 0.002 & 17.005 & 0.002 & 17.392 & 0.001 & 18.783 & 0.002 \\
\hline SDSSJ203722.169-051302.964 & 18.262 & 0.004 & 18.549 & 0.004 & 18.947 & 0.005 & 19.377 & 0.003 & 19.677 & 0.005 & 20.981 & 0.020 \\
\hline SDSSJ210150.65-054550.9 & 18.075 & 0.003 & 18.337 & 0.003 & 18.655 & 0.003 & 19.063 & 0.002 & 19.419 & 0.003 & 20.741 & 0.006 \\
\hline SDSSJ232941.330+001107.755 & 17.947 & 0.004 & 18.110 & 0.004 & 18.161 & 0.004 & 18.473 & 0.003 & 18.784 & 0.004 & 19.993 & 0.006 \\
\hline SDSSJ235144.29+375542.6 & 17.456 & 0.003 & 17.666 & 0.003 & 18.074 & 0.002 & 18.461 & 0.002 & 18.790 & 0.002 & 20.069 & 0.004 \\
\hline \multicolumn{13}{|c|}{ ILAPH } \\
\hline Offsets & $\ldots$ & $\ldots$ & -0.033 & 0.003 & -0.009 & 0.004 & -0.014 & 0.002 & 0.009 & 0.004 & -0.012 & 0.005 \\
\hline G191B2B & 10.490 & 0.001 & 10.890 & 0.001 & 11.499 & 0.001 & 12.031 & 0.001 & 12.451 & 0.001 & 13.885 & 0.002 \\
\hline GD71 & 11.989 & 0.001 & 12.336 & 0.001 & 12.799 & 0.001 & 13.279 & 0.001 & 13.672 & 0.001 & 15.068 & 0.002 \\
\hline GD153 & 12.201 & 0.002 & 12.568 & 0.001 & 13.100 & 0.002 & 13.598 & 0.001 & 14.002 & 0.001 & 15.414 & 0.002 \\
\hline SDSSJ010322.19-002047.7 & 18.195 & 0.004 & 18.527 & 0.005 & 19.083 & 0.005 & 19.569 & 0.005 & 19.965 & 0.006 & 21.355 & 0.012 \\
\hline SDSSJ022817.16-082716.4 & 19.518 & 0.008 & 19.715 & 0.010 & 19.815 & 0.007 & 20.169 & 0.007 & 20.501 & 0.006 & 21.737 & 0.017 \\
\hline SDSSJ024854.96+334548.3 & 17.828 & 0.003 & 18.040 & 0.006 & 18.370 & 0.003 & 18.746 & 0.002 & 19.077 & 0.002 & 20.340 & 0.006 \\
\hline SDSSJ041053.632-063027.580 & 18.116 & 0.009 & 18.404 & 0.004 & 18.879 & 0.005 & 19.254 & 0.003 & 19.393 & 0.005 & 19.498 & 0.005 \\
\hline WD0554-165 & 16.776 & 0.005 & 17.153 & 0.003 & 17.727 & 0.005 & 18.220 & 0.002 & 18.617 & 0.005 & 20.043 & 0.007 \\
\hline SDSSJ072752.76+321416.1 & 17.163 & 0.003 & 17.471 & 0.003 & 17.993 & 0.003 & 18.457 & 0.002 & 18.837 & 0.003 & 20.217 & 0.007 \\
\hline SDSSJ081508.78+073145.7 & 18.950 & 0.006 & 19.263 & 0.008 & 19.716 & 0.005 & 20.184 & 0.005 & 20.579 & 0.006 & 21.962 & 0.024 \\
\hline SDSSJ102430.93-003207.0 & 18.261 & 0.018 & 18.514 & 0.004 & 18.904 & 0.004 & 19.317 & 0.004 & 19.665 & 0.010 & 20.990 & 0.013 \\
\hline SDSSJ111059.42-170954.2 & 17.041 & 0.003 & 17.354 & 0.004 & 17.867 & 0.003 & 18.313 & 0.002 & 18.689 & 0.002 & 20.057 & 0.005 \\
\hline SDSSJ111127.30+395628.0 & 17.443 & 0.004 & 17.830 & 0.006 & 18.420 & 0.003 & 18.939 & 0.004 & 19.344 & 0.002 & 20.797 & 0.009 \\
\hline SDSSJ120650.504+020143.810 & 18.240 & 0.004 & 18.489 & 0.004 & 18.672 & 0.004 & 19.060 & 0.003 & 19.411 & 0.007 & 20.703 & 0.008 \\
\hline SDSSJ121405.11+453818.5 & 16.940 & 0.002 & 17.283 & 0.002 & 17.761 & 0.002 & 18.236 & 0.003 & 18.629 & 0.002 & 20.038 & 0.004 \\
\hline SDSSJ130234.43+101238.9 & 16.188 & 0.002 & 16.522 & 0.002 & 17.036 & 0.002 & 17.514 & 0.002 & 17.904 & 0.002 & 19.303 & 0.004 \\
\hline SDSSJ131445.050-031415.588 & 18.258 & 0.004 & 18.597 & 0.005 & 19.102 & 0.005 & 19.567 & 0.005 & 19.955 & 0.009 & 21.328 & 0.012 \\
\hline SDSSJ151421.27+004752.8 & 15.110 & 0.002 & 15.391 & 0.002 & 15.709 & 0.002 & 16.120 & 0.002 & 16.471 & 0.001 & 17.787 & 0.004 \\
\hline SDSSJ155745.40+554609.7 & 16.500 & 0.002 & 16.877 & 0.002 & 17.470 & 0.003 & 17.992 & 0.002 & 18.388 & 0.002 & 19.834 & 0.005 \\
\hline SDSSJ163800.360+004717.822 & 18.016 & 0.007 & 18.318 & 0.004 & 18.840 & 0.005 & 19.281 & 0.003 & 19.660 & 0.005 & 20.996 & 0.009 \\
\hline SDSSJ172135.97+294016.0 & 20.371 & 0.013 & 20.078 & 0.015 & 19.656 & 0.004 & 19.670 & 0.003 & 19.768 & 0.003 & 20.552 & 0.021 \\
\hline SDSSJ181424.075+785403.048 & 15.791 & 0.002 & 16.121 & 0.002 & 16.544 & 0.002 & 17.001 & 0.002 & 17.393 & 0.001 & 18.786 & 0.002 \\
\hline SDSSJ203722.169-051302.964 & 18.257 & 0.007 & 18.544 & 0.004 & 18.943 & 0.006 & 19.350 & 0.012 & 19.672 & 0.010 & 20.979 & 0.023 \\
\hline SDSSJ210150.65-054550.9 & 18.068 & 0.004 & 18.334 & 0.004 & 18.656 & 0.003 & 19.064 & 0.002 & 19.414 & 0.004 & 20.740 & 0.008 \\
\hline SDSSJ232941.330+001107.755 & 17.943 & 0.004 & 18.109 & 0.004 & 18.161 & 0.006 & 18.470 & 0.003 & 18.775 & 0.007 & 19.995 & 0.006 \\
\hline SDSSJ235144.29+375542.6 & 17.449 & 0.004 & 17.662 & 0.003 & 18.075 & 0.003 & 18.459 & 0.003 & 18.787 & 0.002 & 20.075 & 0.004 \\
\hline
\end{tabular}

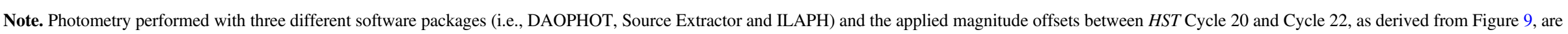
listed. See text for more details.

(This table is available in machine-readable form.) 
CALSPEC standards obtained by using the three different software packages: DAOPHOT, SExtractor and ILAPH. The magnitudes have an average dispersion in the range 1-3 millimag for WFC3-UVIS filters and 5-10 milli-mag for the F160W IR filter. Machine readable photometry is available in Table 9.

Synthetic magnitudes in different photometric systems, such as PS, GAIA, and SDSS, for the set of standard DAWDs will be calculated and provided in NA19.

This study was supported by NASA through grants GO12967 and GO-13711 from the National Optical Astronomy Observatory, which is operated by AURA, Inc., and grant GO15113 from the Space Telescope Science Institute, which is operated by AURA, Inc., under NASA contract NAS 5-26555. E.O. was also partially supported by the NSF through grants AST-1313006 and AST-1815767. This work has made use of data from the European Space Agency (ESA) mission Gaia (https://www.cosmos.esa.int/gaia), processed by the Gaia Data Processing and Analysis Consortium (DPAC, https:// www.cosmos.esa.int/web/gaia/dpac/consortium). Funding for the DPAC has been provided by national institutions, particularly the institutions participating in the Gaia Multilateral Agreement.

Facilities: HST (WFC3), GEMINI, GAIA, Pan-STARRS.

\section{Appendix}

We provide here Table 10, which contains a list and descriptions of all the acronyms used in the manuscript.

Table 10

List of Acronyms used in the Manuscript, Grouped by Class and in Alphabetical Order

\begin{tabular}{ll}
\hline \hline Acronym & Meaning \\
\hline & \multicolumn{1}{c}{ Instruments/Detectors } \\
\hline ACS & Advanced Camera for Surveys \\
GALEX & Galaxy Evolution Explorer \\
GMOS & Gemini Multi-Object Spectrograph \\
HST & Hubble Space Telescope \\
LCO & Las Cumbres Observatory \\
LSST & Large Synoptic Survey Telescope \\
MMT & Multiple Mirror Telescope \\
MSX & Midcourse Space Experiment \\
STIS & Space Telescope Imaging spectrograph \\
UVIS1/2 & Chips of the Wide-Field Camera 3 detector \\
WFC3-IR & Wide-Field Camera 3 Infrared detector \\
WFC3-UVIS & Wide-Field Camera 3 Ultraviolet and VISual detector \\
WISE & Wide-field Infrared Survey Explorer \\
\hline & $\quad$ Surveys \\
\hline ASAS-SN & All Sky Automated Survey for SuperNovae \\
ATLAS & Asteroid Terrestrial-impact Last Alert System \\
DES & Dark Energy Survey \\
PS & Pan-STARSS \\
SDSS & Sloan Digital Sky Survey \\
ZTF & Zwicky Transient Factory \\
\hline & \multicolumn{1}{c}{ Software packages } \\
\hline ALLFRAME & Routine to perform simultaneous point-spread function \\
DAOPHOT & DAOPHOTIV on different images (Stetson 1994) \\
& point-spread function photometry (Stetson 1987) \\
&
\end{tabular}

Table 10

(Continued)

\begin{tabular}{|c|c|}
\hline Acronym & Meaning \\
\hline Drizzle Pac & $\begin{array}{l}\text { Software to stack images collected with Hubble Space } \\
\text { Telescope }\end{array}$ \\
\hline ILAPH & $\begin{array}{l}\text { IDL routines to perform aperture photometry from } \\
\text { Abhijit Saha }\end{array}$ \\
\hline SExtractor & $\begin{array}{l}\text { Source Extractor software to perform aperture and point- } \\
\text { spread function photometry (Bertin \& Arnouts 1996) }\end{array}$ \\
\hline \multicolumn{2}{|r|}{ Others } \\
\hline AS & Artificial star \\
\hline cal_wf3 & $\begin{array}{l}\text { Image calibration pipeline for the Wide-Field Camera } 3 \\
\text { detectors }\end{array}$ \\
\hline CALSPEC & $\begin{array}{l}\text { Database of the Hubble Space Telescope spectro- } \\
\text { photometric standard stars }\end{array}$ \\
\hline CTE & Charge transfer efficiency \\
\hline $\mathrm{CR}$ & Cosmic ray \\
\hline CRNL & Count-rate nonlinearity \\
\hline DAWD & Hydrogen atmosphere white dwarf \\
\hline FoV & Field of view \\
\hline FLUXCORR & $\begin{array}{l}\text { Image header keyword indicating if the flux scaling } \\
\text { needs to be performed }\end{array}$ \\
\hline FWHM & Full-width half maximum \\
\hline IMPHTTAB & Image photometry reference table \\
\hline IR-FIX & $\begin{array}{l}\text { Fixed aperture centered on the Wide-Field Camera } 3 \\
\text { Infrared detector }\end{array}$ \\
\hline IRSUB256-FIX & $\begin{array}{l}256 \times 256 \text { pixel sub-aperture on the center of the Wide- } \\
\text { Field Camera } 3 \text { Infrared detector }\end{array}$ \\
\hline IRSUB512-FIX & $\begin{array}{l}512 \times 512 \text { pixel sub-aperture on the center of the Wide- } \\
\text { Field Camera } 3 \text { Infrared detector }\end{array}$ \\
\hline MODTRAN & MODerate resolution atmospheric TRANsmission \\
\hline NIR & Near-infrared \\
\hline PAM & Pixel area map \\
\hline PHOTFLAM & Image header keyword for the inverse sensitivity \\
\hline PSF & Point-spread function \\
\hline SED & Spectral energy distribution \\
\hline UV & Ultraviolet \\
\hline UVIS1-FIX & $\begin{array}{l}\text { Fixed aperture centered on the UVIS1 chip of the Wide- } \\
\text { Field Camera } 3 \text { Ultraviolet and VISual detector }\end{array}$ \\
\hline $\begin{array}{l}\text { UVIS2- } \\
\text { C512C-SUB }\end{array}$ & $\begin{array}{l}512 \times 512 \text { pixel sub-aperture on the corner of the } \\
\text { UVIS } 2 \text { chip of the Wide-Field Camera } 3 \text { Ultraviolet } \\
\text { and VISual detector }\end{array}$ \\
\hline $\mathrm{ZP}$ & Zero-point \\
\hline
\end{tabular}

\section{ORCID iDs}

Annalisa Calamida (1) https://orcid.org/0000-0002-0882-7702 Thomas Matheson (1) https://orcid.org/0000-0001-6685-0479 Abhijit Saha (1) https://orcid.org/0000-0002-6839-4881 Gautham Narayan (ii) https://orcid.org/0000-0001-6022-0484 Tim Axelrod (i) https://orcid.org/0000-0002-5722-7199 Ralph Bohlin (1) https://orcid.org/0000-0001-9806-0551 Christopher W. Stubbs (1) https://orcid.org/0000-00030347-1724

Susana Deustua (1) https://orcid.org/0000-0003-2823-360X Elena Sabbi (1) https://orcid.org/0000-0003-2954-7643

\section{References}

Adelman-McCarthy, J. K., Agüeros, M. A., Allam, S. S., et al. 2008, ApJS, 175,297

Bertin, E., \& Arnouts, S. 1996, A\&AS, 117, 393

Betoule, M., Kessler, R., Guy, J., et al. 2014, A\&A, 568, A22 
Bohlin, R. C. 2000, AJ, 120, 437

Bohlin, R. C. 2007, in ASP Conf. Ser. 364, The Future of Photometric, Spectrophotometric and Polarimetric Standardization, ed. C. Sterken (San Francisco, CA: ASP), 315

Bohlin, R. C. 2014, AJ, 147, 127

Bohlin, R. C., Colina, L., \& Finley, D. S. 1995, AJ, 110, 1316

Bohlin, R. C., \& Gilliland, R. L. 2004, AJ, 127, 3508

Bohlin, R. C., Gordon, K. D., \& Tremblay, P.-E. 2014, PASP, 126, 711

Brinkworth, C. S., Burleigh, M. R., Lawrie, K., Marsh, T. R., \& Knigge, C. 2013, ApJ, 773, 47

Brinkworth, C. S., Burleigh, M. R., Wynn, G. A., \& Marsh, T. R. 2004, MNRAS, 348, L33

Burke, D. L., Saha, A., Claver, J., et al. 2014, AJ, 147, 19

Chambers, K. C., Magnier, E. A., Metcalfe, N., et al. 2016, arXiv:1612.05560

Deustua, S. E., Mack, J., Bajaj, V., \& Khandrika, H. 2017, WFC3/UVIS Updated 2017 Chip-dependent Inverse Sensitivity Values, Tech. Rep. ISR WFC3-2017-14

Deustua, S. E., Mack, J., Bowers, A. S., et al. 2016, UVIS 2.0 Chip-dependent Inverse Sensitivity Values, Tech. Rep. ISR WFC3-2016-03

Dupuis, J., Chayer, P., Vennes, S., Christian, D. J., \& Kruk, J. W. 2000, ApJ, 537, 977

Filippenko, A. V. 1982, PASP, 94, 715

Flewelling, H. A., Magnier, E. A., Chambers, K. C., et al. 2016, arXiv:1612. 05243

Fontaine, G., \& Brassard, P. 2008, PASP, 120, 1043

Fukugita, M., Ichikawa, T., Gunn, J. E., et al. 1996, AJ, 111, 1748

Gaia Collaboration, Brown, A. G. A., Vallenari, A., et al. 2018, A\&A, 616, 1

Gennaro, M., Bajaj, V., \& Long, K. 2018, A characterization of persistence at short times in the WFC3/IR detector, Tech. Rep. ISR WFC3-2018-05

Gianninas, A., Bergeron, P., \& Ruiz, M. T. 2011, ApJ, 743, 138

Girven, J., Steeghs, D., Heber, U., et al. 2012, MNRAS, 425, 1013

Gorecki, A., Abate, A., Ansari, R., et al. 2014, A\&A, 561, A128

Hayes, D. S., Pasinetti, L. E., \& Philip, A. G. D. (ed.) 1985, IAU Symp. 111 Calibration of Fundamental Stellar Quantities (Dordrecht: Reidel)

Hermes, J. J., Gänsicke, B. T., Gentile Fusillo, N. P., et al. 2017, MNRAS, 468, 1946

Hilbert, B. 2009, WFC3 SMOV Program 11427: UVIS Channel Shutter Shading, Tech. Rep. ISR WFC3-2009-25

Holberg, J. B., \& Bergeron, P. 2006, AJ, 132, 1221

Holberg, J. B., Bergeron, P., \& Gianninas, A. 2008, AJ, 135, 1239

Holberg, J. B., \& Howell, S. B. 2011, AJ, 142, 62

Hook, I. M., Jørgensen, I., Allington-Smith, J. R., et al. 2004, PASP, 116, 425
Horne, K. 1986, PASP, 98, 609

Khandrika, H., Deustua, S., \& Mack, J. 2018, WFC3/UVIS-Temporal and Spatial Variations in Photometry, Tech. Rep. ISR WFC3-2018-16

Kleinman, S. J., Kepler, S. O., Koester, D., et al. 2013, ApJS, 204, 5

Long, K. S., Baggett, S., \& MacKenty, J. W. 2013, Characterizing Persistence in the WFC3 Channel: Observations of Omega Cen, Tech. Rep. ISR WFC32013-07

Long, K. S., Wheeler, T., \& Bushouse, H. 2011, IR Detector Timing and Persistence, Tech. Rep. ISR WFC3-2011-09

Massey, P., \& Gronwall, C. 1990, ApJ, 358, 344

Massey, P., Strobel, K., Barnes, J. V., \& Anderson, E. 1988, ApJ, 328, 315

Matheson, T., Kirshner, R. P., Challis, P., et al. 2008, AJ, 135, 1598

McCook, G. P., \& Sion, E. M. 1999, ApJS, 121, 1

Narayan, G., Axelrod, T., Holberg, J. B., et al. 2016, ApJ, 822, 67

Narayan, G., Matheson, T., Saha, A., et al. 2019, ApJS, in press (arXiv:1811. 12534)

Oke, J. B. 1974, ApJS, 27, 21

Oke, J. B., \& Gunn, J. E. 1983, ApJ, 266, 713

Rauch, T., Werner, K., Bohlin, R., \& Kruk, J. W. 2013, A\&A, 560, A106

Riess, A. G. 2010, First On-orbit Measurements of the WFC3-IR Count-rate NonLinearity, Tech. Rep. ISR WFC3-2010-07

Riess, A. G. 2011, An Independent Determination of WFC3-IR Zeropoints and Count Rate NonLinearity from 2MASS Asterisms, Tech. Rep. ISR WFC32011-15

Riess, A. G., \& Petro, L. 2010, Boosting Count-rates with Earth Limb Light and the WFC3/IR Count-rate Nonlinearity, Tech. Rep. ISR WFC3-2010-15

Sabbi, E. 2009, WFC3 SMOV Program 11798: UVIS PSF Core Modulation, Tech. Rep. ISR WFC3-2009-20

Sahu, K., Baggett, S., \& MacKenty, J. 2014, Use of the Shutter Blade Side for UVIS Short Exposures, Tech. Rep. ISR WFC3-2014-09

Sahu, K., Gosmeyer, C. M., \& Baggett, S. 2015, WFC3/UVIS Shutter Characterization, Tech. Rep. ISR WFC3-2015-12

Schmidt, G. D., Weymann, R. J., \& Foltz, C. B. 1989, PASP, 101, 713

Scolnic, D., Casertano, S., Riess, A., et al. 2015, ApJ, 815, 117

Shanahan, C. E., Gosmeyer, C. M., \& Baggett, S. 2017, 2017 Update on the WFC3/UVIS Stability and Contamination Monitor, Tech. Rep. ISR WFC32017-15

Stetson, P. B. 1987, PASP, 99, 191

Stetson, P. B. 1994, PASP, 106, 250

Stone, R. P. S. 1977, ApJ, 218, 767

Stubbs, C. W., \& Brown, Y. J. 2015, MPLA, 30, 1530030

Welch, D. L., \& Stetson, P. B. 1993, AJ, 105, 1813 